\title{
Observation of fast stochastic ion heating by drift waves
}

\author{
J. M. McChesney, a) P. M. Bellan, and R. A. Stern ${ }^{\text {) }}$ \\ California Institute of Technology, Pasadena, California 91125
}

(Received 30 March 1990; accepted 16 August 1991)

\begin{abstract}
Anomalously fast ion heating has been observed in the Caltech Encore tokamak [Phys. Rev. Lett. 59, 1436 (1987) ], with the use of laser-induced fluorescence. This heating was found to be independent of electron temperature, but was well correlated with the presence of largeamplitude drift-Alfvén waves. Evidence is presented that suggests that the heating is stochastic and occurs when the ion displacement due to polarization drift becomes comparable to the perpendicular wavelength, i.e., when $k_{\perp}\left(m_{i} k_{1} \phi_{0} / q B^{2}\right) \sim 1$. Stochastic heating may also be the cause of the anomalously high ion temperatures observed in reversed-field pinches.
\end{abstract}

\section{INTRODUCTION}

In this paper, we describe the first application of laserinduced fluorescence (LIF) techniques to the majority ions of a tokamak. The application of LIF to plasma ions was first proposed by Measures' and Dimock et al. ${ }^{2}$ The technique was first successfully applied to plasmas by Stern and Johnson, ${ }^{3}$ who used LIF to obtain ion velocity distribution functions in argon plasmas. The technique has previously been applied to tokamak plasmas; however, in those experiments the majority hydrogen or deuterium ions were fully stripped and only hydrogen neutrals (e.g., Burakov et al. ${ }^{4}$ ) or impurity ions (e.g., Muller and Burrell ${ }^{5}$ ) could be studied. The present series of experiments were performed in the Encore high repetition tokamak, ${ }^{6}$ which produced hydrogen or noble gas plasmas. Spectroscopic studies revealed that the argon plasmas produced in Encore were chiefly singly ionized. Using a tunable narrow band laser, it was possible to trace out ion velocity distributions in ArII plasmas. Over a wide range of experimental conditions, ion heating in Encore was anomalously fast, i.e., much faster than expected through classical collisional energy exchange with the hot electrons. The anomalous heating and high ion temperatures were not strongly correlated with electron temperatures, but were correlated with the presence of low-frequency $\left(\omega<\omega_{c i}\right)$ drift-Alfvén waves. In the present work we show how ion motion can become stochastic, in the presence of such modes, resulting in bulk ion heating. Using a one-dimensional slab model, it is shown that stochastic heating is possible when the drift wave potential fluctuation amplitude exceeds a threshold value given by $\phi_{0}=q B_{0}^{2} / m_{i} k_{\perp}^{2}$. This condition corresponds to the breakdown of the guiding center approximation and occurs when the ion displacement caused by the polarization drift becomes comparable to the wavelength of the mode. Experimental evidence is presented that supports the proposed heating mechanism. Preliminary results of this work have presented previously; ${ }^{\circ}$ in this paper we give a more complete discussion and also present several additional points.

The application of the "optical tagging" technique to

\footnotetext{
" Present address: General Atomics, P. O. Box 85608, San Diego, California 92186-9784.

b) Permanent address: University of Colorado, Boulder, Boulder, Colorado 80309.
}

the more complicated tokamak geometry is described. This technique allows the transport of small groups of ions to be determined. ${ }^{7}$ The technique is used to demonstrate gyrorotation of ions in Encore. We also present an interesting speculation regarding the application of this heating mechanism to ion heating in reversed-field pinches (RFP's) and fieldreversed configuration devices (FRC's). Spectroscopic and charge exchange analyses of RFP's have shown ion temperatures in excess of the electron temperature and rates of ion heating far exceeding those predicted by Ohmic heating. ${ }^{8,9}$ Plasma conditions in RFP's are similar to those in Encore. In particular, several important dimensionless ratios are identical, leading us to speculate that drift waves are present in RFP's and that they are responsible for the fast heating observed in these devices.

\section{APPARATUS}

The laser-induced fluorescence measurements were performed on the Caltech Encore tokamak device. Several features make Encore attractive for fluorescence work. It is typically operated using noble gas discharges, allowing fluorescence experiments to be performed on noble gas ions. It can also be operated using hydrogen, although this was not done in the present work. It is a high repetition rate device (typically 15 discharges per second) allowing signal averaging to be employed. This high repetition rate is obtained by using a dc toroidal field and a high power, audio-frequency amplifier to drive the Ohmic heating transformer. Typical Encore parameters are $R=38.1 \mathrm{~cm}, a=12.6 \mathrm{~cm}, B=0.1-$ $1.5 \mathrm{kG}, I_{p}=0.05-10 \mathrm{kA}, n_{e} \simeq 10^{12} \mathrm{~cm}^{-3}, T_{i}=1-5 \mathrm{eV}$, $T_{e}=10-20 \mathrm{eV}$, and pulse length $=1-5 \mathrm{msec}$. Because of the large spacing between the toroidal field coils, access was excellent. A specially constructed chamber section permitted virtually the entire poloidal cross section to be interrogated. Lateral stabilization of the plasma was provided by the 1.27 $\mathrm{cm}$ thick aluminum walls and by a vertical field. Energy and particle confinement times were low in Encore. For an input power of $5 \mathrm{~kW}$, typical plasma parameters are $n_{c}=10^{12}$ $\mathrm{cm}^{-3}, T_{e}=15 \mathrm{eV}$, and $T_{i}=1 \mathrm{eV}$, giving an energy confinement time of $50 \mu \mathrm{sec}$. Assuming Bohm diffusion and a magnetic field of $300 \mathrm{G}$ gives a confinement time of $\tau_{\mathbf{B}} \sim 250$ $\mu$ sec. Line radiation is evidently the main cause of energy loss. Summers and $\mathrm{McWhirter}{ }^{10}$ give tables of radiation power-loss coefficients for a variety of different plasmas. 
These tables confirm that, for the parameters above, argon plasmas can radiate $5-10 \mathrm{~kW}$ of power.

The laser system used in the present work was a narrow band, tunable dye laser (Lambda Physik model FL 2001) pumped by a copper vapor laser (Plasma Kinetics model 451). Coarse tuning of the dye laser was performed using a prism beam expander and grating with a resolution of 0.2 $\mathrm{cm}^{-1}$. The linewidth of the dye laser was narrowed by means of an intracavity Fabry-Perot etalon. The specified resolution of the system $0.035 \mathrm{~cm}^{-1}$. The dye laser output was calibrated by means of an external Fabry-Perot interferometer (Spectra Physics model 410-G2, free spectral range $=29.5 \mathrm{GHz}$ ). Using this etalon the output linewidth was found to be $1.3 \mathrm{GHz}$ (corresponding to an ion temperature resolution of $0.047 \mathrm{eV}$ in argon), and computer-controlled tuning was found to be accurate to within a few percent. Unstable resonator optics were used to reduce the beam divergence from 10 to $1 \mathrm{mrad}$. With the unstable resonator the threshold for laser action was about $60 \mathrm{~kW}$ of pump power. The laser dye selected for the present work was Kiton Red 620. This dye has a lasing maximum at $620 \mathrm{~nm}$ and a useful wavelength range of 595-639 $\mathrm{nm}$ when pumped by a copper vapor laser. The absorption maximum is at 554 $\mathrm{nm}$, which is conveniently placed between the two copper vapor laser pump lines at 510 and $578 \mathrm{~nm}$. A small amount of Rhodamine 640 was added to the Kiton Red 620 to improve pumping efficiency in the region $610-620 \mathrm{~nm}$. Dye concentrations were $0.4 \mathrm{gl}^{-1}$ (Kiton Red) and $0.1 \mathrm{gl}^{-1}$ (Rhodamine). The maximum dye laser efficiency using this solution was $10 \%$. This was reduced to $5 \%$ when the intracavity etalon was inserted.

The layout of the apparatus is shown in Fig. 1. The probing laser beam was directed vertically into the plasma by means of a movable mirror. The fluorescence signal induced by this beam was viewed perpendicular to the beam direction by a converging lens $(f=15 \mathrm{~cm})$ and photomultiplier tube (PMT). An interference filter (linewidth $1 \mathrm{~nm}$ ) centered on the fluorescence line was used to remove all but the desired line. A $1 \mathrm{~mm}$ diam pinhole served to limit the light incident on the PMT to acceptable levels and at the same time set the spatial resolution. The lens/PMT tube combination was also movable, allowing the entire shaded region shown in Fig. 1 to be interrogated. The translators were driven by computercontrolled stepper motors. Finally, a polarization rotator and a polarizer were used to polarize the laser beam parallel to the toroidal field direction. This was done to minimize the effect of Zeeman splittings, which were, in any case, small.

\section{LASER-INDUCED FLUORESCENCE}

The laser-induced fluorescence (LIF) technique is based upon the selective excitation of an atomic transition by the absorption of laser radiation of the appropriate wavelength. The chief attraction of this method is its high sensitivity, which is due to the large cross section for photoexcitation $\left(\approx \lambda^{2} / 8 \pi \sim 10^{-10} \mathrm{~cm}^{2}\right)$. $^{3}$ This value is roughly 14 orders of magnitude larger than that for Thomson scattering. Once in the upper level, an atom or ion may decay via a different transition, emitting a photon having a different wavelength from the absorbed photon. This emission will, of

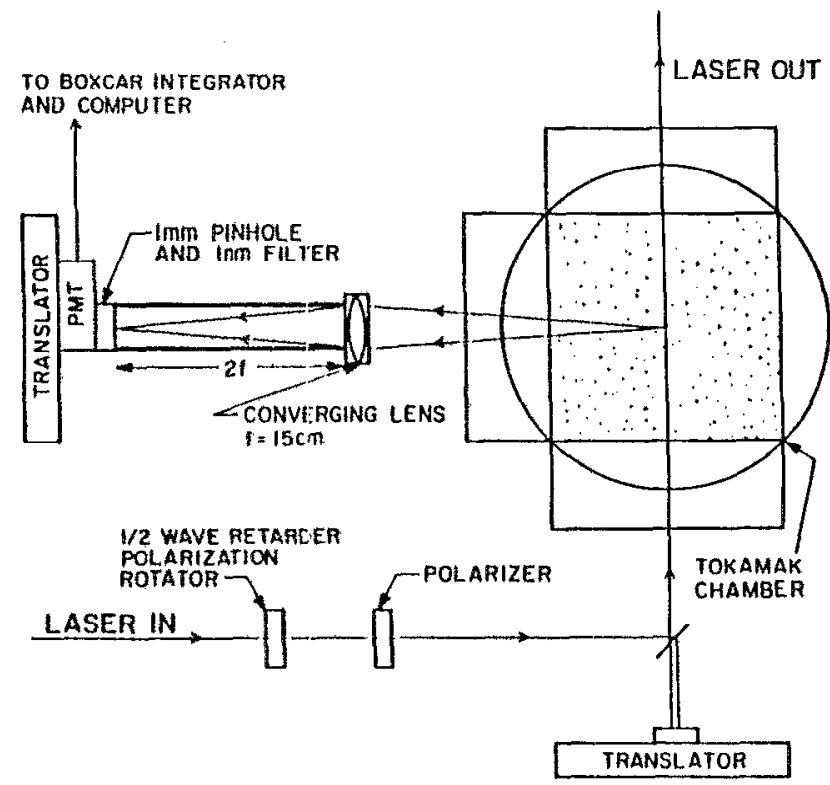

FIG. 1. Schematic showing the experimental layout.

course, originate from the region along the laser path. By viewing the emitted radiation perpendicular to the beam direction, the diagnosed volume will be determined by the intersection of the field of view of the viewing optics and the beam path.

In principle, LIF is capable of measuring virtually all plasma parameters of interest; (i) ion and atomic velocity distribution functions (hence, ion and atomic temperatures) and densities, ${ }^{3}$ ion and atomic particle trajectories, ${ }^{7}$ electron temperatures from the relative populations of the excited levels, the effective ion charge and the local electric field by means of the Stark effect, " the vector direction and magnitude of the local magnetic field by means of the Zeeman effect. ${ }^{12}$ In the presert work, only techniques for (i) and (ii) will be discussed. Other examples of the application of LIF in laboratory and fusion plasmas include the work of Koslover and $\mathrm{McW}$ Williams ${ }^{13}$ (multidimensional, ion velocity distributions by optical tomography); Burakov et al. ${ }^{4}$ (local hydrogen densities in a tokamak); Muller and Burrell ${ }^{5}$ (impurity ion densities in a tokamak). The LIF method does have limitations. It cannot be applied to $\mathrm{H}^{+}$or other fully stripped ions, which immediately limits the use of the technique in fusion reactors. Strong background radiation may mask the emitted fluorescence radiation. In high-density plasmas, the plasma may become optically thick and absorb the fluorescence signal. Finally, the technique is confined to the visible and near ultraviolet regions of the spectrum, where convenient, tunable radiation sources are available.

Two examples of ArII fluorescence schemes are shown in Fig. 2. For these schemes, the fluorescence lines are different from the exciting laser lines. The scheme selected for most of the present work was that shown in Fig. 2(a). These schemes were particularly convenient because the excitation line was in the red region of the spectrum, where several efficient laser dyes can be used. In addition, the fluorescence 

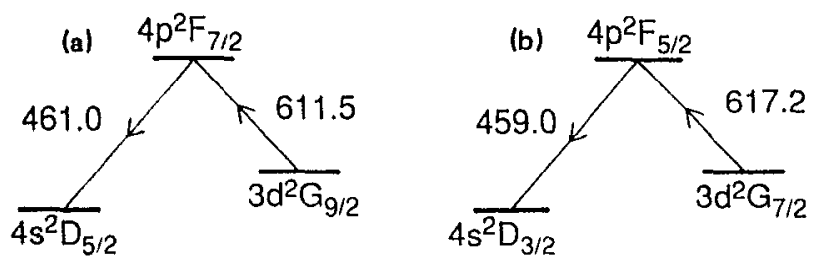

FIG. 2. Examples of fluorescence schemes in ArII.

lines were in the blue, where PMT's are particularly efficient. In both schemes shown in Fig. 2, the target state is an elevated metastable state (not the ground state), allowing visible laser radiation to be used. The ion velocity distribution function was measured by scanning the narrow band dye laser across the Doppler-broadened absorption line. It is important to ensure that all line broadening mechanisms other than Doppler broadening are negligible, or can be corrected for. This will also be discussed in the next paragraph.

In the low-density plasmas obtained in Encore, Doppler broadening is the dominant line-broadening mechanism. The Doppler-broadened intensity distribution function takes the form

$I_{\mathrm{D}}(\lambda)=\sqrt{\frac{m_{i} c^{2}}{2 \pi \kappa T_{i} \lambda_{0}^{2}}} \exp \left(\frac{-m_{i} c^{2}\left(\lambda-\lambda_{0}\right)^{2}}{2 \kappa T_{i} \lambda_{0}^{2}}\right)$,

where the symbols have their usual meanings. The Doppler width for a $10 \mathrm{eV}$ argon plasma is $\sim 2 \times 10^{-2} \mathrm{~nm}$. For comparison the natural linewidth is $\sim 10^{-4} \mathrm{~nm}$, i.e., completely negligible. Owing to the low plasma densities in the Encore device, collisional Stark broadening is also negligible. The only other broadening mechanisms that are important in Encore are instrumental in nature, and are caused by the finite linewidth of the laser. The laser linewidth was measured to be $1.3 \mathrm{GHz}$. The observed Doppler lineshape was corrected for instrumental broadening during data reduction. If two statistically independent, line-broadening mechanisms resulting in lineshapes of $I_{1}(\Delta \lambda)$ and $I_{2}(\Delta \lambda)$, respectively, are simultaneously at work, then the according to Griem, ${ }^{14}$ the resultant lineshape is given by the convolution of these lineshapes, $I_{\text {obs }}(\Delta \lambda)=\int I_{1}\left(\Delta \lambda-\Delta \lambda^{\prime}\right)$ $\times I_{2}\left(\Delta \lambda^{\prime}\right) d \Delta \lambda^{\prime}$. For the present case, $I_{2}$ is a Gaussian of the form given in Eq. ( 1 ), and $I_{1}$ is assumed to be a Fabry-Perot "comb" function. Ion temperatures were found iteratively by minimizing the least-squares difference between the measured distribution and the calculated integral for $I_{\text {obs }}$. Another possible instrumental effect is power (or saturation) broadening, which occurs when the stimulated photon emission rate equals the photon absorption rate and both are greater than the spontaneous photon emission rate. When saturation occurs, the power residing in the wings of the laser line becomes important, resulting in photon absorption far from the laser line center and therefore extra line broadening. An experimental check on the effects of power broadening was made by progressively reducing the laser intensity by means of neutral density filters and measuring the Doppler lineshape at each stage. This experiment was per- formed at $T_{i} \sim 1.5 \mathrm{eV}$. No power-broadening effects were observed at this temperature. The maximum measured laser intensity at the plasma position was $100-200 \mathrm{~W} \mathrm{~cm}^{-2}$, and it is possible that power broadening was significant for the lowest temperature measurements $\left(T_{i}<0.2 \mathrm{eV}\right)$. Finally, the possible effects of Zeeman splitting are considered. Zeeman shifts are of the order of $\Delta \lambda \simeq 10^{-11} \lambda^{2} B$, where the wavelengths are in nanometers and the magnetic field is in Gauss. For $B=1 \mathrm{kG}$ and $\lambda=600 \mathrm{~nm}$ the shift is $3.6 \times 10^{-3}$ $\mathrm{nm}$, resulting in a slight broadening of the observed Dopplerbroadened distribution. However, by aligning the polarization of the probing laser beam along the toroidal magnetic field direction only the $\pi$ components were excited. The splittings of the individual $\pi$ components are much smaller than the observed Doppler broadening, so the Zeeman effect was not important in our work.

Argon plasmas were used exclusively in the present work. Such plasmas were found to be particularly reproducible, and several convenient LIF schemes were possible in singly ionized argon plasmas. Spectroscopic studies indicated that argon plasmas in Encore were predominantly singly ionized. No lines corresponding to ArI, ArIV, or higher charge states were observed. Some Arin lines were observed, but they were of considerably lower intensity than the ArII lines. Figure 3 shows the relative intensities of various ArII and ArII lines roughly $1.5 \mathrm{msec}$ into a typical discharge, where the plasma current was $1 \mathrm{kA}$. These spectra were measured using a $0.5 \mathrm{~m}$ monochrometer, which was scanned between 185 and $900 \mathrm{~nm}$. The output of the monochrometer, which was fed into a boxcar averager, allowing the relative intensities of the various lines to be measured at fixed times into the discharge. For the lower plasma currents used in the present work, the ratio of ArIII to ArrI line intensity was smaller than indicated by Fig. 3. If coronal equilibrium were assumed a dominant charge state of $Z=4-5$ would be predicted. The explanation for the predominance of ArII lies in

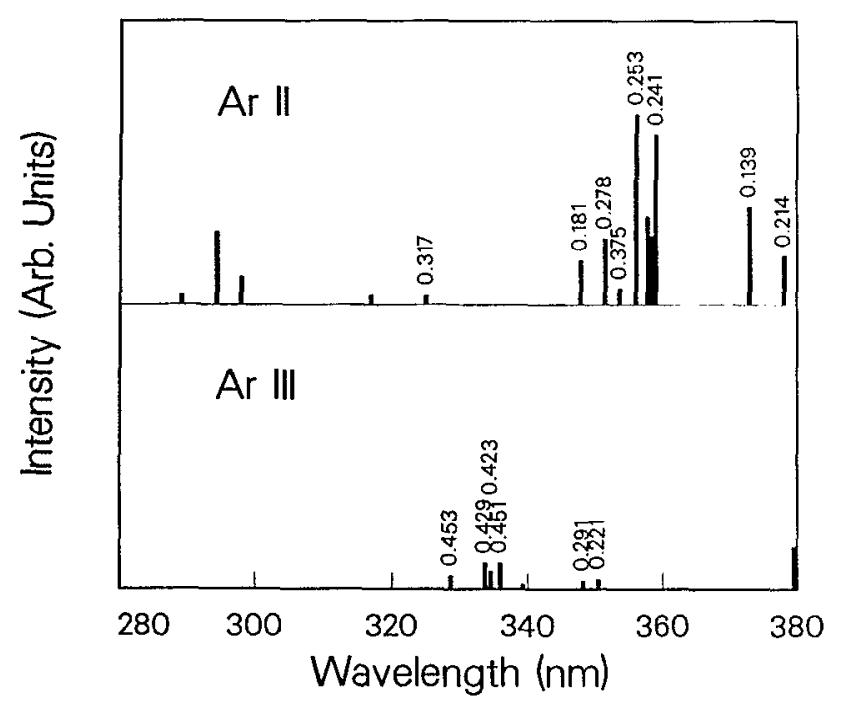

FIG. 3. Relative intensities (arbitrary units) of ArII and ArII lines in the range 320-380 $\mathrm{nm}$ for a typical Encore discharge. The plasma current was 1 $\mathrm{kA}$ and the field strength was $350 \mathrm{G}$. Oscillator strengths are shown for selected lines. 
the relative ionization rates of the various charge states. The ionization rate coefficients can be calculated for each state using the semiempirical Lotz formulas. ${ }^{15}$ It was found that the rate coefficient for each successive charge state was about ten times higher than that for the previous state. Radiative and three-body recombination rates are both negligible. Thus, ArI was rapidly ionized while ArI was ionized much more slowly and ArrI more slowly still. The simultaneous ion loss rates were quite high, and the Arm ions were lost before they could be further ionized. Assuming an ion loss time of $250 \mu \mathrm{sec}$, it was straightforward to solve the coupled rate equations for the first few ionization states of argon on a computer, and hence calculate the densities of these states. Figure 4 plots the results of these calculations, along with $Z_{\text {eff }}$. The ArI density decreases to a low level and the ArI increases to its maximum level within the first 100 $\mu$ sec. After about $300 \mu \mathrm{sec}$ the ArII density equals the ArII density, but $Z_{\text {eff }}$ remains well below 2 at all times. The populations of the higher charge states are negligible.

\section{OPTICAL TAGGING}

Optical tagging is an extension of the LIF technique that allows the transport of small groups of ions to be determined. ${ }^{7}$ It requires a multilevel quantum system with at least one long-lived state. To demonstrate the technique, consider the fluorescence scheme shown in Fig. 2(a). To perform tagging the population of the $3 d^{2} G_{9 / 2}$ state is depleted by means of a pump laser tuned to the $3 d^{2} G_{9 / 2} \rightarrow 4 p^{2} F_{7 / 2}$ transition. Because of the high transition probability for the downward $4 p^{2} F_{7 / 2} \rightarrow 4 s^{2} D_{5 / 2}$ transition, many ions will end up in the $4 s^{2} D_{5 / 2}$ state. Suppose that some or all of these ions are transported away from the position of the pump laser. If a second search laser is now tuned to the $3 d^{2} G_{9 / 2} \rightarrow 4 p^{2} F_{7 / 2}$ transition (but at a different position to the pump laser), the presence of tagged ions at the position of the search laser will

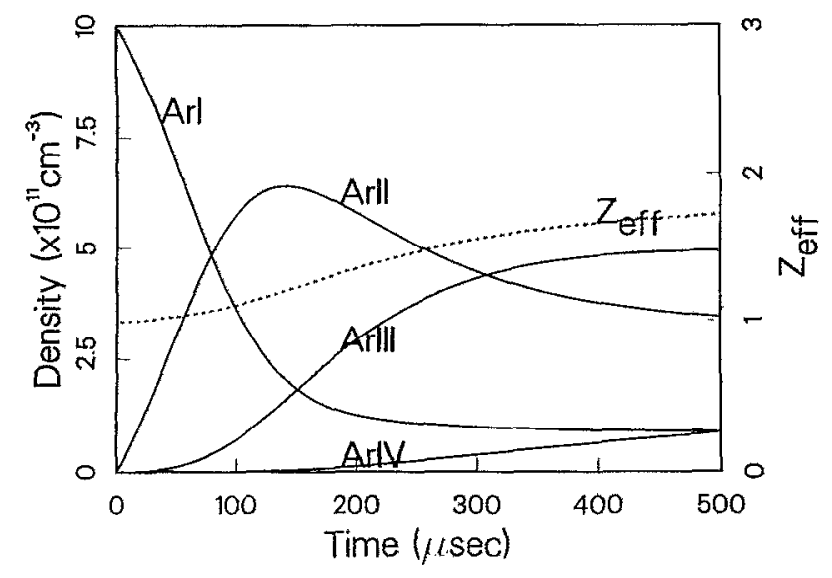

FIG. 4. Calculations showing the relative densities of ArI, ArII, ArIn, and Arrv as a function of time. Also plotted is $Z_{\text {er }}$. Parameters used were $n_{\text {neur }}$ $=1 \times 10^{12} \mathrm{~cm}^{-3}, n_{c}=2 \times 10^{11} \mathrm{~cm}^{-3}$, and $T_{c}=12 \mathrm{eV}$. reduce the fluorescence signal, since the density of target states has been reduced. This reduction in the fluorescence signal reflects the transport from the position of the pump laser to the position of the search laser. The utility of tagging is limited by the liferime of the metastable target states concerned. Electron excitation serves to return metastable populations to their steady state levels. The $3 d^{2} G_{9 / 2}$ level is radiatively connected to several levels, and these levels will all contribute to the repopulation of this particular level and decrease the tagging lifetime. The lifetime of the $3 d^{2} G_{9 / 2}$ tagged state was experimentally measured, and was typically 5-10 $\mu \mathrm{sec}$. Since ion thermal velocities in Encore were of the order of $1-3 \times 10^{5} \mathrm{cn} \mathrm{sec}^{-1}$, this short lifetime made it difficult to observe transport over distances greater than a 1-2 $\mathrm{cm}$. It is possible tc inject fast ions in order to apply this technique to the study of ion transport over greater distances.

Optical tagging can be used to perform velocity selection perpendicular to the direction of the probing laser beams. If two pulsed laser beams are spatially separated by a distance $d$ much greater than their diameters and are temporally separated by a period $t$ much longer than their duration, then, only those ions moving from the pump beam to the search beam with an average velocity of $d / t$ will contribute to the reduction or enhancement of the search beam fluorescence. If it is assumed that the ions perform perfect gyromotion, i.e., their orbits are perfectly circular or helical, then only a small subclass of ions can interact with both beams. Such ions must posses both a perpendicular velocity $\left(v_{1}\right)$ and an initial phase $\left(\phi_{0}\right)$, which satisfy the relation

$$
d=\left(v_{1} / \omega_{c i}\right)\left[\sin \left(\omega_{c i} t+\phi_{0}\right)-\sin \left(\phi_{0}\right)\right] .
$$

Refer to Fig. 5(a) for the geometry involved in this problem. If the search beam is narrow band, it may be scanned to perform velocity selection parallel to the beam direction. The only tagged lons that can now interact resonantly with the beam are those whose $y$ components of velocity are given by $v_{y}=v_{\perp} \sin \left(\omega_{c i} t+\phi_{0}\right)$, where $v_{\perp}$ and $\phi_{0}$ satisfy Eq. (2). Assuming that the initial phase $\phi_{0}$ is completely random and that the ions assume a Maxwellian distribution, it is possible to calculate the lineshape of the tagged ion velocity distribution. Using the following experimental parameters; $d=1$ $\mathrm{cm}, t=3 \mu \mathrm{sec}, B_{0}=1400 \mathrm{G}$, and $T_{i}=1.5 \mathrm{eV}$, the results shown in Fig. 5(b) were obtained. Also plotted in this figure is the Maxwellian ion distribution used to derive the tagged function. The resulting distribution is no longer Maxwellian and its peak has been shifted away from zero. Reversing the direction of gyrorotation reverses the direction of this shift. To scan only the tagged ions, the following procedure was adopted. The search $(S)$ laser was scanned across the Doppler-broadened line on a discharge to discharge basis, but the pump $(P)$ laser was fired only every alternate search laser discharge. In this manner, it was possible to obtain simultaneously the usual Doppler-broadened distribution function and the distribution function that contains the tagged component. By subtracting these (using a two-channel boxcar integrator with alternately triggered channels), it was possible to obtian the distribution function for the tagged component alone. This experiment was then repeated 


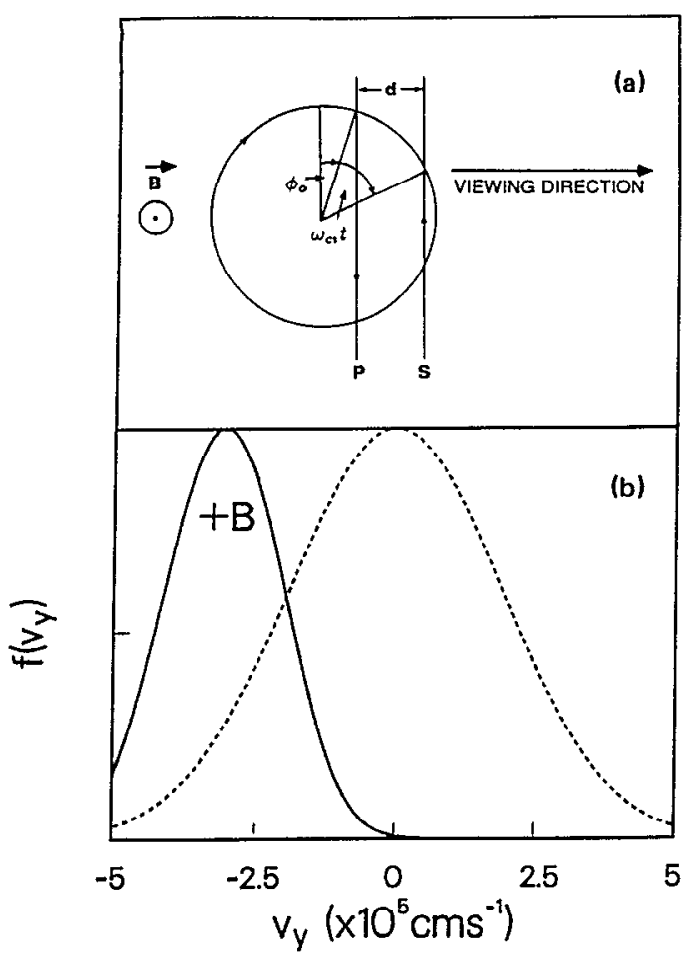

FIG. 5. (a) Geometry of the "tagging" experiment used to demonstrate gyrorotation, showing " $P$ " and " $S$ " beams and the Larmor orbit of an arbitrary ion. (b) Calculated lineshape for normal Doppler-broadened line (dashed) and for tagged ion components (solid), assuming $d=1 \mathrm{~cm}, t=3$ $\mu \mathrm{sec}, B=1400 \mathrm{G}$, and $T_{1}=1.5 \mathrm{eV}$.

with the toroidal magnetic field reversed. Figure 6 shows the results of this experiment for two different beam separations, $1 \mathrm{~mm}$ and $4 \mathrm{~mm}$, respectively. (The separations here are not the distances between beam centers but the distances between the edges of the two beams.) The temporal separation was $3 \mu \mathrm{sec}$ for both spatial separations and the magnetic field was $1400 \mathrm{G}$. In both instances, the tagged distributions are definitely shifted relative to the untagged Maxwellian. The direction of this shift depends on the toroidal field direction and the magnitude of the shift increases with increasing beam separation. It is evident from Fig. 6 that the distribution functions of the tagged ions were non-Maxwellian, but the quality of the data was not good enough to allow for further analysis of the actual lineshape.

\section{ANOMALOUS ION HEATING IN ENCORE}

The plasma in Encore is Ohmically heated by means of a toroidally directed plasma current. The Ohmic heating process involves the rapid heating of the electrons, followed by the heating of the ions on a time scale slower by a factor of $m_{t} / m_{e}$ ( $\approx 10^{5}$ for argon ions). The classical time for equilibration between electron and ion temperatures is given by Trubnikov, ${ }^{16}$

$$
\tau_{i e}^{E}=\frac{3 \sqrt{2 \pi} \pi \epsilon_{0}^{2}\left(m_{i} \kappa T_{e}+m_{e} \kappa T_{i}\right)^{3 / 2}}{Z^{2} \sqrt{m_{e} m_{i}} n \lambda_{i e} e^{4}},
$$

where the symbols have their usual meanings. For parameters relevant to Encore, $\tau_{i e}^{E} \simeq 20-50 \mathrm{msec}$. Figure 7 (a)

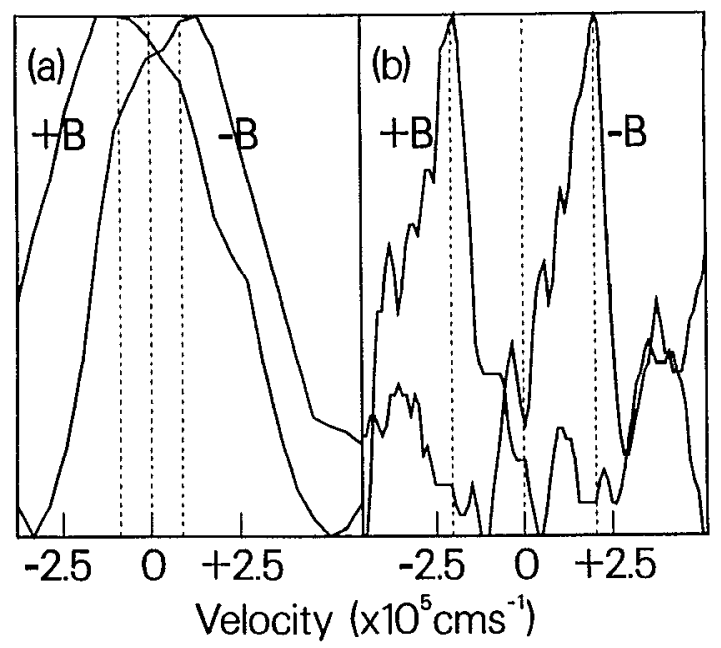

FIG. 6. Ion distribution functions (of tagged ions) showing the effect of magnetic field reversal on the position of the peak. (a) Beam separation is 1 $\mathrm{mm}$. (b) Beam separation is $4 \mathrm{~mm}$.

shows a sequence of ion distribution functions recorded during the first $500 \mu \mathrm{sec}$ of an Encore discharge. The data were taken at the plasma center. Plotted in Fig. 7(b) are ion temperatures $T_{i}$ determined from Gaussian fits to these distribution functions, normalized to the electron temperature $T_{e}$ determined from Langmuir probe measurements. Both $T_{i}$ and $T_{e}$ were measurcd at the center of the plasma. The relatively low heat fluxes incident on the Langmuir probe allowed it to be inserted to the center of the plasma. The surprising feature of Fig. 7 is that the observed rates of ion heating far exceed those predicted by Eq. (3). Ion temperatures reach $\sim 4 \mathrm{eV}$ within 100-200 $\mu \mathrm{sec}$, giving a heating rate of about $2-4 \times 10^{4} \mathrm{eV} \mathrm{sec}^{-1}$. For the electron temperature and density relevant to this discharge, a heating rate of about $350 \mathrm{eV} \mathrm{sec}^{-1}$ is predicted from Eq. (3). It will be

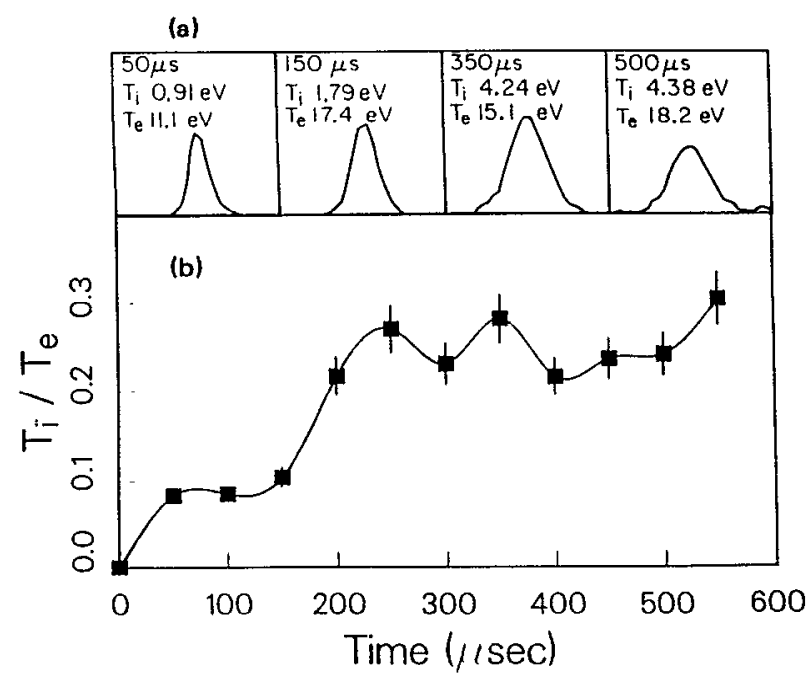

FIG. 7. Experimental demonstration of fast ion heating in Encore. (a) Examples of measured ion distribution functions. (b) $T / / T_{e}$ as a function of time. 
shown that none of the usual ion heating mechanisms is able to explain the observed ion heating.

\section{THEORETICAL BACKGROUND}

\section{A. Particle heating mechanisms}

Particle heating requires that particle motion be irreversible. In a nonturbulent plasma, it is evident that there exist only three means to ensure irreversibility; collisions, resonant wave-particle interactions, and intrinsic stochasticity. Collisions are the chief mechanism in low-temperature or dense plasmas, but collisions amongst like particles cannot increase the aggregate energy of the colliding particles. Resonant wave-particle interactions lead to irreversibility and can also increase the aggregate particle energy (at the expense of wave energy ), however, they do not heat the plasma bulk and require collisions to transfer energy from the resonant particles to the bulk. In contrast, stochastic heating mechanisms can lead to the irreversible transfer of energy to the bulk plasma particles. In Sec. V it was shown that ion heating rate in Encore was about $2-4 \times 10^{4} \mathrm{eV} \mathrm{sec}^{-1}$. It will now be shown that the most obvious "classical" heating mechanisms cannot account for the observed fast heating.

(i) Direct heating by the toroidal electric field $E$. The ion and electron drift velocities $u_{i}, u_{e}$ caused by $E$ are determined by $m_{i} \dot{u}_{i}=e E-m_{i}\left(u_{i}-u_{e}\right) / \tau_{i e}$ and $m_{e} \dot{u}_{e}$ $=-e E-m_{e}\left(u_{e}-u_{i}\right) / \tau_{e i}$, where total momentum conservation gives $m_{i} u_{i}+m_{e} u_{e}=0$ and $\tau_{e i} m_{i}=\tau_{i e} m_{e}$. The steady-state drift velocities are $u_{e}=-e E \tau_{e i} / m_{e}$ and $u_{i}$ $=e E \tau_{e i} / m_{i} \simeq 1200 \mathrm{~cm} \mathrm{sec}^{-1}$ with $\tau_{e i}=3.5 \times 10^{5} T_{e}^{3 / 2} / n \lambda$ $\simeq 1 \mu \mathrm{sec}$, where $n \simeq 10^{12} \mathrm{~cm}^{-3}, \lambda \simeq 15$, and $T_{e} \simeq 12 \mathrm{eV}$. Frictional drag of ions on electrons will give ion heating. The frictional ion heating rate is $d T_{i} / d t=e E^{2} \tau_{e i} m_{i}$ $\simeq 60 \mathrm{eV} \mathrm{sec}^{-1}$. Similarly, the drag due to neutrals is $\left(\sigma_{\text {neut }} n_{\text {neut }} u_{i}\right) m_{i} u_{i} \quad$ giving $d T_{i} / d t=\sigma_{\text {neut }} n_{\text {neut }} u_{i}^{3} m_{i} / e$ $\simeq 5.4 \times 10^{-4} \mathrm{eV} \mathrm{sec}^{-1}\left(\sigma_{\text {neut }} \sim 7.5 \times 10^{-15} \mathrm{~cm}^{2}\right.$ and $n_{\text {neut }}$ $\sim 10^{12} \mathrm{~cm}^{-3}$ ). Clearly, direct heating by the toroidal electric field is insufficient. The neutral density quoted here is estimated using the known neutral fill density of $1-1.7 \times 10^{12}$ $\mathrm{cm}^{-3}$ and assuming a fairly low degree of ionization. The results of Sec. III indicate that the plasma should become highly ionized within $50-100 \mu \mathrm{sec}$, so this value is conservative.

(ii) Equilibration with electrons. In Ohmically heated discharges, the electrons are rapidly heated, while inns are heated more slowly by collisional energy exchange with the hot electrons. Electrons can heat up to $10-15 \mathrm{eV}$ very quickly, $\left(d T_{e} / d t=e E^{2} \tau_{e i} / m_{c} \simeq 4.5 \times 10^{6} \mathrm{eV} \mathrm{sec}^{-1}\right)$, and will then transfer energy to the ions at a rate of $d T_{i} / d t=T_{e} / \tau_{i c}^{E}$ $\simeq 300 \mathrm{eV} \mathrm{sec}^{-1}$. This mechanism is also wholly insufficient to account for the observed ion heating rate of $2-4 \times 10^{4}$ $\mathrm{eV} \mathrm{sec}^{-1}$.

(iii) Scattering by ion-neutral collisions. In weakly ionized plasmas, it has been proposed that the directed $\mathbf{E} \times \mathbf{B}$ drift velocity may be randomized by ion-neutral collisions. ${ }^{17}$ Using the values $\sigma_{\text {neut }} \sim 7.5 \times 10^{-15} \mathrm{~cm}^{2}$ and $n_{\text {neut }} \sim 10^{12}$ $\mathrm{cm}^{-3}$, the mean-free path is found to be about $150 \mathrm{~cm}$, a distance much larger than the minor radius. Therefore this process is unimportant. (iv) Several electrostatic instabilities have been observed to cause ion heating in other devices. These include the ion cyclotron instability (Dakin et al. ${ }^{18}$ ), the ion acoustic instability (Mah et al ${ }^{19}{ }^{1}$ ) and drift waves (Hatakeyama et al. ${ }^{20}$ ). The only instability of significant amplitude observed in Encore is the drift-Alfven instability. This mode will be shown to be responsible for the observed ion heating.

(v) Ion Landau damping on drift waves. During the first $50 \mu \mathrm{sec}$ of the discharge, $T_{i}<1 \mathrm{eV}$ so that the parallel ion velocity $\left(\sim 1 \times 10^{5} \mathrm{~cm} \mathrm{sec}^{-1}\right.$ is much smaller than the parallel phase velocity of the drift-Alfvén wave $\left(\sim 5 \times 10^{6}\right.$ $\mathrm{cm} \mathrm{sec}-1$ ) making Landau damping effects (linear and nonlinear) completely negligible.

(vi) Heating by idrift wave turbulence. This is not possible because heating is also observed when the drift waves are coherent.

\section{B. Drift-Alfvén waves}

Drift waves are microinstabilities driven by the free energy available in the pressure gradient found in all magnetically confined laboratory plasmas. They propagate at the electron diamagnetic drift velocity in a direction almost perpendicular to the magnetic field and to the pressure gradient. Drift waves also propagate parallel to the magnetic field (with $k_{\|} \ll k_{\perp}$ ). Mikhailovskii and Rudakov ${ }^{21}$ predicted the existence of drift waves having substantial magnetic fluctuations when the parallel drift wave velocity becomes comparable to the Alfvén velocity, i.e., $\omega / k_{\|} \sim v_{\mathrm{A}}$. This condition is equivalent to the condition $\beta>m_{e} / m_{i}$, where $\left(\beta=8 \pi n T_{e} / B^{2}\right)$. Fredrickson and Bellan ${ }^{22}$ demonstrated that the drift oscillations observed in Encore were, in fact, drift-Alfvén oscillations. In the Appendix it is shown that the modes observed in Encore are current driven. In the saturated state it is assumed that the mode amplitude is proportional to the growth rate, which, in turn, is proportional to the destabilizing plasma current. Therefore by using very low plasma currents $(<100 \mathrm{~A})$, drift waves in Encore could be stabilized at very low amplitudes. At this point the exact saturation mechanism is unknown, but it is thought to be related to the loss of wave energy to the ions, which are then heated and ejected from the plasma.

The modes observed in Encore could assume different mode structures, depending upon the central safety factor $q(0)$ of the particular discharge. For example, when the toroidal magnetic field strength was about $200-500 \mathrm{G}$ and the plasma current about $0.2 \mathrm{kA}, q(0) \sim 2$, and the electrostatic fluctuations were highly coherent (with toroidal mode number $n=1$, poloidal mode number $m=2$, and frequency $=5$ $\mathrm{kHz}$ ). When the toroidal field was raised, keeping the plasma current constant, $q(0)$ increased, and the electrostatic modes were localized toward the edge of the plasma. As $q(0)$ increased the modes changed from $m=2$ to $m=4$, and then to a more turbulent mixture of higher-order modes. If, instead, the plasma current was increased such that $q(0)<1$, higher-frequency, turbulent fluctuations are observed. Figure 8 shows two ion saturation current traces obtained using a cylindrical Langmuir probe. These plots were taken with a toroidal magnetic field of $300 \mathrm{G}$ and plasma currents of 0.2 and $2 \mathrm{kA}$, respectively. For $0.2 \mathrm{kA}$ the mode was clearly 


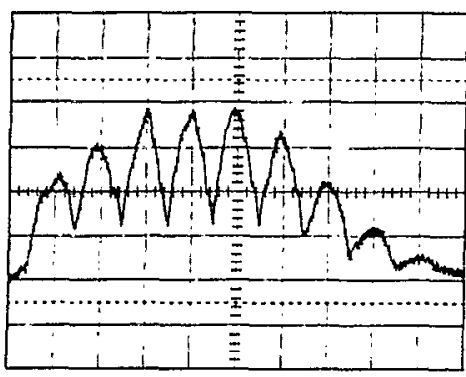

(a)

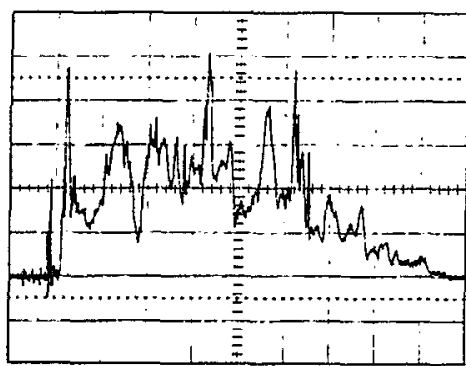

(b)

Hor. Scale $200 \mu \mathrm{s} / \mathrm{div}$

FIG. 8. Ion saturation current traces measured near the plasma edge of (a) coherent $m=2$ drift waves and (b) turbulent drift waves.

highly coherent (with toroidal mode number $n=1$, poloidal mode number $m=2$, and frequency $=5.0 \mathrm{kHz}$ ), while for 2 kA the mode structure was more turbulent. Figure 9 shows contour plots of density, electron temperature, space potential, and vector potential of the coherent $m=2$ mode. The poloidal mode structure is clearly evident from the plots. These plots were made using Langmuir probes and magnetic loop probes. ${ }^{22}$ From the space potential plot it can be seen that the drift waves generate very large electric fields $\left(k_{\perp}\right.$ $\sim 30 \mathrm{~m}^{-1}, \phi_{0} \sim 2 \mathrm{~V} \Rightarrow E_{1}=k_{1} \phi_{0} \simeq 60 \mathrm{~V} \mathrm{~m}^{-1}$ ). Such fields are much larger than the toroidal electric field due to the Ohmic heating transformer $\left(E_{\|} \simeq 5 \mathrm{~V} \mathrm{~m}^{-1}\right)$.

Strong ion heating was associated with the presence of both coherent and turbulent drift waves. In the rest of the present work heating by the coherent variety of drift waves will be emphasized, because it was possible to accurately measure the plasma potential fluctuations of these coherent waves by means of Langmuir probes. This was not the case for the turbulent variety of drift wave. However, the essential ion heating mechanism was felt to be the same both cases.

\section{Stochastic ion motion in a drift-Alfven wave}

The importance of Hamiltonian stochasticity in classical systems has long been recognized. Several theoretical papers have predicted that the motion of a single magnetized ion may become stochastic in the field of an electrostatic wave (e.g., Smith and Kaufman ${ }^{23}$ and Karney ${ }^{24}$ ). Particularly pertinent to the present work is the study by Drake and Lee $^{25}$ of stochastic heating of electrons by low-frequency lower hybrid drift waves. Experimentally, stochastic electron motion has been observed in a standing plasma wave by Doveil ${ }^{26}$ and Skiff $e t a l .{ }^{27}$ have reported stochastic ion heating in the field of a neutralized ion Bernstein wave. Cheng and Okuda ${ }^{28}$ have demonstrated ion heating by drift waves using computer simulations but did not identify the heating as being stochastic.

Stochastic heating takes place when particle motion becomes sufficiently chaotic that large regions of phase space can be traversed by the particles. The conditions under which this can occur are investigated by considering a slab model of an ion moving in the potential of an electrostatic drift wave $(O \rightarrow y, r \rightarrow x, m / r \rightarrow k)$. The fields are described by

$$
\phi=-\phi_{0} \sin (k y-\omega t), \quad \mathbf{B}=B_{0} \hat{z} .
$$

The Lorentz equation describing the ion motion is

$$
m_{i} \frac{d \mathbf{v}}{d t}=q\left[k \phi_{0} \hat{y} \cos (k y-\omega t)+\mathbf{v} \times B_{0} \hat{z}\right] .
$$

When $\omega \ll \omega_{c i}$, Eq. (5) is usually solved using the guidingcenter approximation, $\mathbf{v}=\mathbf{v}_{E \times B}+\mathbf{v}_{\text {pol }}$, where, $\mathbf{v}_{\mathrm{pol}}$ $=\dot{\mathbf{E}} / \omega_{c i} B_{0}$. In this approximation it is assumed that $\dot{\mathrm{v}}_{\mathrm{pol}} \ll \dot{\mathrm{v}}_{E \times B}$. Substituting the expression for the electric field into the expression for $\mathbf{v}_{\text {pol }}$ yields

$$
v_{y}=\frac{m_{i}}{q B_{0}^{2}}\left(\frac{\partial E_{y}}{\partial t}+v_{y} \frac{\partial E_{y}}{\partial y}\right) .
$$

The solution for $v_{y}$ will diverge as

$$
\alpha=m_{i} k^{2} \phi_{0} / q B_{0}^{2} \rightarrow 1 \text {. }
$$

The polarization drift is in the direction of the wave phase, while the $\mathbf{E} \times \mathbf{B}$ drift moves the ion perpendicular to this direction. From Eq. (6) it can be seen that when $\alpha \geqslant 1$, the assumption that $\dot{\mathbf{v}}_{\text {pol }} \ll \dot{\mathbf{v}}_{E \times B}$ breaks down and the guidingcenter approximation is no longer valid, and the ion motion must be calculated exactly. From Eq. (7), $\alpha \sim k \Delta y$, where $\Delta y$ is the ion displacement due to the polarization drift, so the condition $\alpha \sim 1$ implies that $\Delta y$ becomes comparable to the drift wave perpendicular wavelength. It is also apparent that the above notions apply only to electrostatic modes where $\mathbf{E}|| \mathbf{k}$; hence, $\mathbf{v}_{\mathrm{pol}} \| \mathbf{k}$ and the polarization drift can push ions into regions of different wave phase. For purely electro-
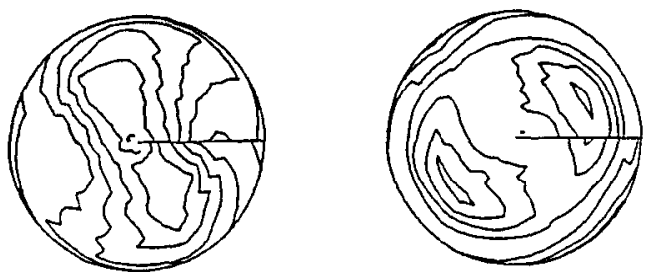

(a) DENSITY $\left(5 \times 10^{11} \mathrm{~cm}^{-3} /\right.$ contour $)$

(b) ELECTRON TEMP. $(2.5 \mathrm{cV} /$ contour $)$
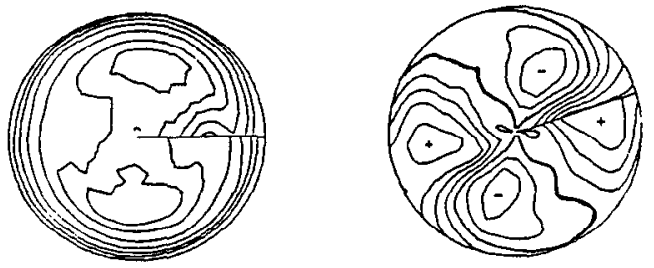

(c) SPACE POTENTIAL (2.5 V/conten, (d) VECTOR P'O'TENTIAL, (1 stat wolt/contonr)

FIG. 9. Contour plots of (a) density, (b) electron temperature, (c) space potential, and (d) vector potential. Plots are those of Fredrickson and Bellan. ${ }^{22}$ 
magnetic waves, $\mathbf{E} \perp \mathbf{k}$ and the polarization drift cannot do this. The above reasoning can be generalized to two dimensions, where the potential is assumed to be a traveling wave in the $y$ direction, and a standing wave in the $x$ direction. For simplicity it is assumed that $k_{z}=k_{y}$;

$$
\phi=-\phi_{0} \sin (k x) \sin (k y-\omega t), \quad \mathbf{B}=B_{0} \hat{z} .
$$

Again assuming guiding-center motion, the polarization drift velocity can be written as

$$
\begin{aligned}
& v_{x}=\frac{m_{i}}{q B_{0}^{2}}\left(\frac{\partial E_{x}}{\partial t}+v_{x} \frac{\partial E_{x}}{\partial x}+v_{y} \frac{\partial E_{x}}{\partial y}\right), \\
& v_{y}=\frac{m_{i}}{q B_{0}^{2}}\left(\frac{\partial E_{y}}{\partial t}+v_{x} \frac{\partial E_{y}}{\partial x}+v_{y} \frac{\partial E_{y}}{\partial y}\right) .
\end{aligned}
$$

Substituting the expression for the electric potential into these two linear equations in $v_{x}$ and $v_{y}$, yields the following determinant:

$$
\operatorname{det}=\left[1+\left(m_{i} k^{2} \phi / q B_{0}^{2}\right)\right]\left[1+\left(m_{i} k^{2} \phi / q B_{0}^{2}\right)\right] .
$$

Again the determinant diverges as $\alpha \rightarrow 1$, however, since there are now two ways in which the determinant can diverge, it is reasonable to suppose that the motion will become nonadiabatic more quickly than for the one-dimensional case. Typically, for the present experiments, $\omega / 2 \pi=5 \mathrm{kHz}$, $k=m / r=33 \mathrm{~m}^{-1}(m=2, r=0.06 \mathrm{~m}), \phi_{0}=2 \mathrm{~V}$, and $B_{0}=0.03 \mathrm{~T}$, giving $v=0.44$ and $\alpha=1.0$, so that stochastic ion heating should occur.

The conditions necessary for stochastic ion motion can be found numerically by constructing surface of section plots. For the sake of simplicity, the following discussion refers to the one-dimensional electric field described by Eq. (4). Normalizing times to $\omega_{c i}^{-1}$, lengths to $k^{-1}$, and writing $v=\omega / \omega_{c i}$, Eq. (5) becomes,

$$
\ddot{y}+y=\alpha \cos (y-v t), \quad \dot{x}=y .
$$

Numerically integrating Eq. (12) gave the ion trajectory in the phase space $\dot{y}, y, v t$. A three-dimensional phase space must be used to describe the ion motion and such a system is said to have $\frac{3}{2}$ degrees of freedom. Such a system has the interesting property of being the lowest-dimensional system that is capable of exhibiting stochastic behavior. Figure 10 shows a series of surface of section plots for increasing values of $\alpha$ when $v=0.4767$. The different orbits shown in each plot represent different initial positions of the test ion in the wave. Each test ion trajectory was followed for several hundred cyclotron periods. For small values of $\alpha$, the orbits all lie on smooth curves and are integrable. For intermediate values of $\alpha$, some of the orbits may be stochastic, depending on the initial position. For $\alpha>0.8$, however, essentially all orbits for $|\dot{y}|<3$ are stochastic, and ions may be heated through the range of phase space $|\dot{y}|<3$. The same behavior was observed for $v$ down to 0.05 . Clearly, when $v=0$, however, the Lorentz equation [Eq. (12)] becomes integrable, and ion motion cannot become stochastic. The surface of section plots show a threshold for stochastic heating. From Fig. 10(f) it can be seen that stochastic ions may be accelerated to velocities of $\dot{y}>3$. By constructing a series of surface of section plots for $\alpha>0.8$, it was found that the maximum velocity to which an ion can be accelerated is given by $\dot{y}_{\max }$ $\approx \alpha+2.3$. The maximum velocity that an ion can attain in a stationary wave potential is $\dot{y}_{\max }=2 \sqrt{\alpha}$. Therefore the ratio of maximum stochastic energy to maximum nonstochastic energy is

$$
H_{\text {stochastic }} / H_{\text {nonstcchastic }} \approx(\alpha+2.3)^{2} / 4 \alpha .
$$

So, very high ion energies can be obtained by increasing the wave amplitude $\alpha$. In particular, ions can be accelerated to energies in excess of the electrostatic potential energy of the "well." Note that for $\alpha \sim 0.8$, the energy ratio given by Eq. (13) is about 3, so there should be significant numbers of ions having kinetic energies greater than the "well" potential energy.

When $\nu \ll 1$ the stochastic behavior described above is caused by the trapping and detrapping of ions in the periodic, potential troughs of the drift waves. The Hamiltonian describing ion motion is

$h=\left(1 / 2 m_{i}\right)\left[\left(p_{x}+q B_{0} y\right)^{2}+p_{y}^{2}\right]-q \phi_{(y} \sin (k y-\omega t)$,

where $p_{x}, p_{y}$ are the canonical momenta in the $x, y$ directions. Normalizing as before, Eq. (14) may be rewritten as

$$
h=\frac{1}{2} p_{y}^{2}+\Phi,
$$

where the effective potential $\Phi$ is defined by

$$
\Phi=\frac{1}{2}\left(p_{x}+y\right)^{2}-\alpha \sin (y-v t) .
$$

From Eq. (15) note that $p_{x}=-(y-\dot{x})$ is the negative of the $y$ component of the guiding center and without loss of generality may be taken to be zero, giving

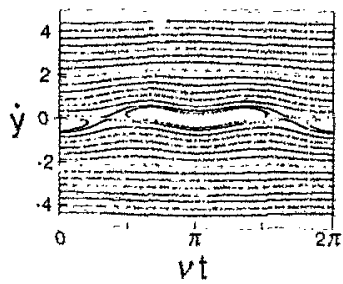

(a)

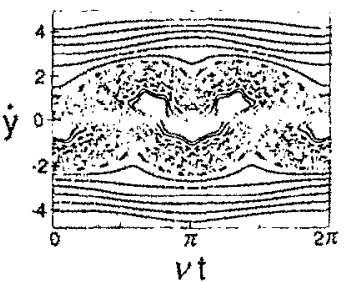

(d)

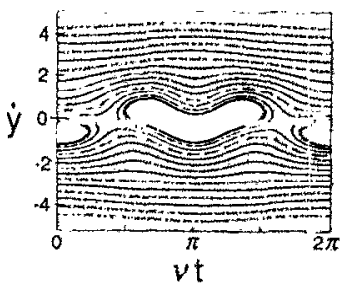

(b)

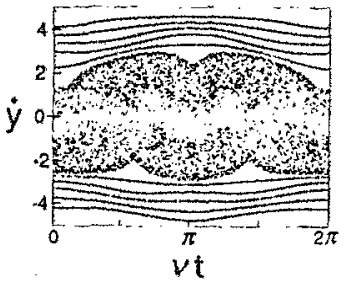

(e)

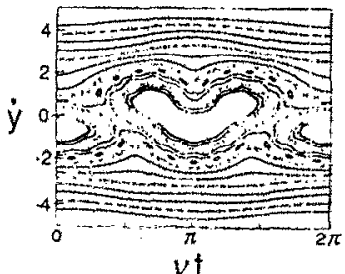

(c)

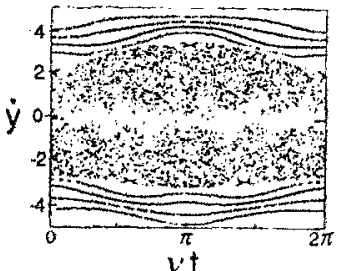

(f)

FIG. 10. Surface of section plots in $(\dot{y}, y, v t)$ space defined by $y=0$ $v=0.4667$. (a) $\alpha=0.2$, (b) $\alpha=0.4$, (c) $\alpha=0.6$, (d) $\alpha=0.7$, (e) $\alpha=0.8$, and (f) $\alpha=1.0$. 


$$
\Phi=\frac{1}{2} y^{2}-\alpha \sin (y-y t) .
$$

The first term in Eq. (17) represents the magnetic part of the effective potential, while the second term represents the electrostatic potential that is due to the drift wave. Following Chen and Birdsall, ${ }^{29} \mathrm{Eq}$. (17) may be represented graphically, as shown in Fig. 11. The initial guiding-center position is at $y=0$. If $T_{i}$ is the initial ion kinetic energy, then the ion Larmor radius is given by the intersection of the $T_{i}$ energy level with the parabola $y^{2} / 2$. For convenience, the wave trough is initially assumed to be at the origin. In order for trapping to occur $\Phi$ must have a local maximum at some point, i.e.,

$$
\frac{\partial^{2} \Phi}{\partial y^{2}}<0 \Rightarrow \alpha>1
$$

As the wave travels from left to right, the ion is carried along by the wave until, at a point of inflection in $\Phi$, the wave electric force equals the magnetic $\mathbf{v} \times \mathbf{B}_{0}$ force, and the ion can be untrapped, i.e.,

$$
\frac{\partial \Phi}{\partial y}=0, \quad \frac{\partial^{2} \Phi}{\partial y^{2}}=0 \Rightarrow y=\sqrt{\alpha^{2}-1} .
$$

The time for which the ion experiences the trapping force is given by

$$
\tau=y / v=\sqrt{\alpha^{2}-1} / v .
$$

If $\alpha=1.5$ and the wave frequency is $5 \mathrm{kHz}$, then this time will be $\sim 35 \mu \mathrm{sec}$. At the point where it is untrapped the ion will have a potential energy of $\left(\alpha^{2}+1\right) / 2$. The maximum energy that it can attain by falling into the potential well is given by the difference between this energy and the minimum possible potential energy, $\Phi_{\min }=-\alpha$ at the bottom of the electrostatic well, i.e., $(\alpha+1)^{2} / 2$. Therefore the maximum velocity that an ion can achieve is bounded and that boundary is defined by $\dot{y}_{\max }=\alpha+1$. The most energetic ions are found at the bottom of the potential well, where $y=0$ and $v t=\pi$. On average, ions will fall into the well minima at $\Phi \sim 0$ and, $\dot{y}_{\text {avg }} \simeq \sqrt{\alpha^{2}+1}$. In unnormalized units,

$$
T_{i}=m_{i}\left[\left(k \phi_{0} / B_{0}\right)^{2}+\left(\omega_{c i} / k\right)^{2}\right] \text {. }
$$

In other words $v_{T i} \gtrsim v_{E \times B}$. These points are clearly demonstrated in Fig. 11, where the large value for $\alpha$ was selected in order to make the cffects more obvious. The above arguments are only exact when $v \ll 1$ and the above results will be altered when the wave phase velocity is finite. In the present work and the work of Drake and Lee, ${ }^{25}$ low-frequency waves $\left(\omega<\omega_{c \prime}\right)$ were considered and the underlying physical mechanism causing stochastic behavior was particle trapping. In Karney's work, ${ }^{24}$ high-frequency waves were considered $\left(\omega \gg \omega_{c i}\right)$ and ion motion became stochastic as a result of the nonlinear interaction of the resonances between the cyclotron motion and the wave. If the interacting of field is of sufficient amplitude, the ion "forgets its phase" relative to the wave during a cyclotron period. At some point in their orbits such ions will experience the wave-particle resonance condition $\omega=\mathbf{k} \cdot \mathbf{v}$.

At this point it is perhaps appropriate to emphasize the importance of the concept of intrinsic stochasticity. The conditions necessary for stochastic heating to occur are modest in the sense that any combination of electric and magnetic

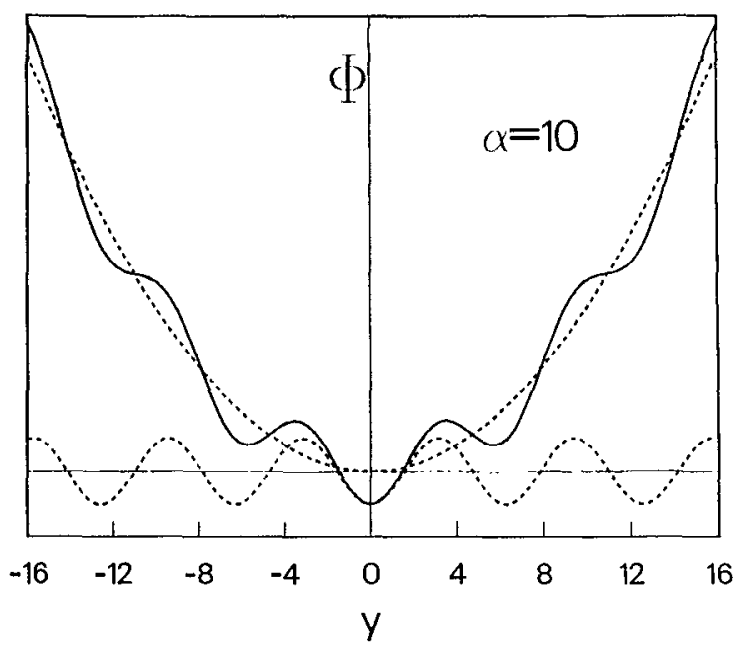

FIG. 11. Effective potential seen by an ion. The effective potential is the solid curve. The magnetic and electrostatic potentials are the dashed curves. The sinusoidal wave propagates from left to right.

fields that predicts stochastic behavior theoretically will unquestionably yield stochastic behavior experimentally. Quoting Chernikov et al.," "Stochasticity has a number of amusing and interesting properties. We are accustomed to the fact that various solutions which are 'good' in simple modes, are spoiled when more complex models are adopted. The situation changes, however, in the region of stochastic dynamics. Further complications of the model lead to an increase in the phase volume occupied by the stochastic region. Hence to discover the condition for the onset of stochasticity, it is sufficient to study the simplest situations." Hence the simple model discussed above is in no sense academic; in fact, it yields necessary and sufficient conditions to ensure stochastic ion behavior.

\section{EXPERIMENTAL VERIFICATION OF STOCHASTIC ION HEATING}

\section{A. Introduction}

In this section several pieces of evidence supporting stochastic acceleration of ions will be presented. First, the Landau damping of ion acoustic waves will be examined. It will be shown that theoretical damping lengths calculated using LIF determined ion temperatures were in excellent agreement with those observed experimentally. For low-amplitude drift waves, ion temperatures were low and Landau damping was experimentally observed to be weak, but as the wave amplitude was increased, $T_{i}$ also increased, and Landau damping increased strongly. Second, it will be shown that the heating was strongest where the mode amplitude is largest, i.e., at the plasma edge. Third, it will be shown that the observed in heating increased strongly above a certain wave amplitude. Fourth, it will be shown that the ion motion was nonconservative, by showing that some groups of ions that were initially cold, attained kinetic energies that exceeded the maximum possible electrostatic potential energies. Finally, the results of a numerical simulation of ion heating in the field of a prescribed electrostatic mode will be shown 


\section{Experiment}
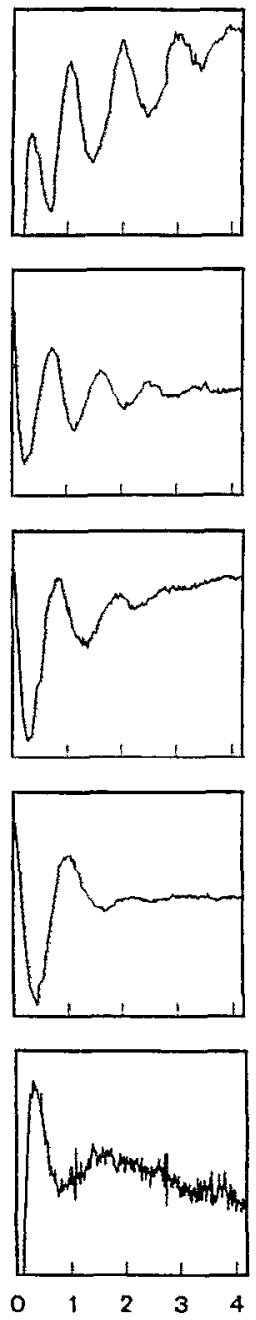

Probe Sep. $(\mathrm{cm})$

FIG. 12. Interferometer traces of Landau-damped ion acoustic waves, showing the effect of increasing $T_{c} / T_{4}$.

to be consistent with experimentally observed ion temperatures and heating rates.

\section{B. Landau damping of ion acoustic waves}

The observation of ion acoustic waves in the Encore device has been difficult. In the course of the present work it became evident that this difficulty stemmed from the unexpectedly high ion temperatures, and hence low ratio of $T_{c} / T$, observed in the Encore device. As is well known, the Landau damping rate increases strongly with decreasing $T_{c} / T_{i}$. The real and imaginary parts of the ion acoustic wave number may be written ${ }^{31}$ as

$k_{r}=\omega / c_{\text {, }}$

$k_{i}=-k_{r} \sqrt{\frac{\pi}{8}}\left[\left(\frac{T_{c}}{T_{i}}\right)^{3 / 2} \exp \left(-\frac{T_{c}}{2 T_{t}}\right)+\sqrt{\frac{m_{c}}{m_{i}}}\right]$, where $c_{s}$ is the ion sound speed. By running very low current plasmas, where the drift waves were not destabilized, and strong ion heating was absent, it was possible to observe ion acoustic waves. Using an interferometer, measurements of wavelength and damping length were made as a function of increasing plasma current. Heterodyne detection was used to improve the signal to noise ratio. Sound waves were excited using a small rectangular plate $(2 \times 1.5 \mathrm{~cm})$ and an $\mathrm{L}$ shaped receiving probe $(3 \mathrm{~mm})$. A wave frequency of 500 $\mathrm{kHz}$ was selected in order to obtain wavelengths of about 1 $\mathrm{cm}$. Such a wavelength is small compared to the plasma radius but large compared to the receiving probe dimensions. In addition, $\omega \gg \omega_{c i}$, enabling the wavelength scans to be made perpendicular to the magnetic field.

The results of this experiment are shown in Fig. 12. The ion sound speed was found from the wavelength and frequency of the acoustic waves, which allowed the electron temperature to be cetermined. Langmuir probe measurements were used to verify the electron temperatures thus determined. The Langmuir probe temperatures were found to be about $10 \%$ higher than those determined from the wavelength measurements. It was suspected that plasma conditions were slightly different when the probe measurements were made. Ion temperature measurements were made using LIF. From the measured values of $T_{e}$ and $T_{i}$, the theoretical damping sould be calculated. The results of these calculations are displayed alongside the experimental data. Incluced in these calculations is a $1 / r$ falloff in wave amplitude with probe separation. The agreement between the two sets of figures is excellent and there is strong damping of the acoustic waves as $T_{c} / T_{i}$ decreases. The agreement is poorer for low $T_{i}$ because the damping length scales as $\lambda \sim\left(T_{i} / T_{e}\right)^{3 / 2} \exp \left(T_{i} / 2 T_{i}\right)$, so that a small absolute error in $T_{i}$ translates to a very large error in the damping length. This effect is less pronounced for larger $T_{i}$.

These data were important because they proved that the ion temperatures determined using LIF were accurate. Additionally, the strong damping of ion acoustic waves implies that the ion distribution functions obtained on a shot-to-shot basis, using LIF, represent true thermalization of the ion velocities, and not ar average over coherent motions.

\section{Radial variation of ion heating}

As was discussed in Sec. VI B, in high toroidal field discharges, where $q(0)>1$, the drift wave activity was localized toward the edge. In addition, the strong hield served to limit the radial transport of hot ions. Hence, in high $q(0)$ discharges, the highest heating rates would be expected to occur at the plasma edge. Such results were, in fact, observed when using a high toroidal field of $1400 \mathrm{G}$ and a plasma current of less than $1 \mathrm{kA}$. In this instance, the drift waves did not possess the highly coherent nature shown in Fig. 8(a) but had the more turbulent nature shown in Fig. 8(b). Figure 13(a) shows ion saturation current traces obtained at two different radial plasma positions, viz., $r / a=0$ and $r / a=0.67$. Figure 13 (b) shows the time evolution of ion temperature at these same positions. During the first $1.5 \mathrm{msec}$ of the discharge, when the relative flustuation levels were low at the plasma 
center but high at the edge, the ion heating rate at the edge was clearly twice that at the center. It was only later in the discharge, when the fluctuations at the center became larger, that the central ion temperatures approached the edge temperatures. This phenomenon was also observed by Hatakeyama et al., ${ }^{20}$ who looked at heating by drift waves in a Qmachine plasma. They, however, used electrostatic energy analyzers to measure ion temperatures and evidently did not elucidate the actual ion heating mechanism. It should be noted that because stochastic acceleration results in diffusion in both velocity and coordinate space, ion temperatures at the center are not zero, and, in fact, eventually come into equilibrium with those at the edge.

\section{Observation of threshold condition}

The surface of section plots shown in Sec. VI indicate a fairly distinct threshold for stochastic heating with increasing mode amplitude. Below a drift wave amplitude corresponding to $\alpha \sim 0.8$, the ion heating rate should be low. This behavior was observed experimentally. Figure 14 shows the peak ion temperature during an Encore discharge as a function of $\alpha$, where $\alpha$ was determined using experimentally measured values of $k_{\theta}, \phi_{0}$, and $B_{0}$. For small $\alpha$, ion temperatures remained below $0.5 \mathrm{eV}$. However, when $\alpha$ was increased beyond 0.4 ion temperatures nearly tripled. This behavior is expected from the theoretical analysis of Sec. VI. For the last point in Fig. 14, $\phi_{0}=1.5 \mathrm{~V}, k_{\theta}=32.0 \mathrm{~m}^{-1}$, $B_{0}=275 \mathrm{G}$, and $T_{i}=1.25 \mathrm{eV}$, giving $\alpha=0.85$. Equation (21) predicts $T_{i}=3 \mathrm{eV}$, a value that was similar to the mea-

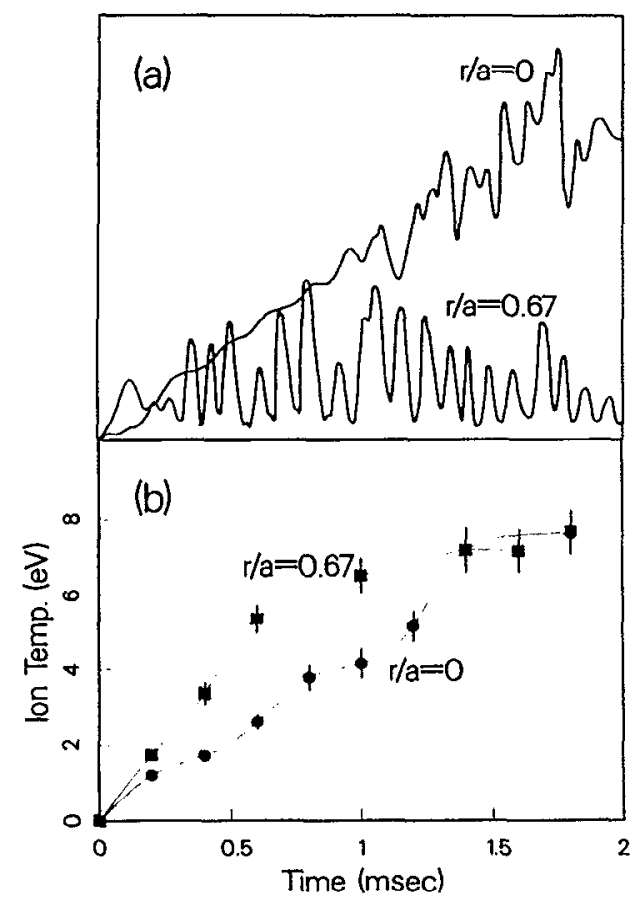

FIG. 13. (a) Ion saturation currents, at two plasma positions $r / a=0$, and $r / a=0.67$. (b) Ion temperatures, at the same positions, showing the correspondence between relative fluctuation level and ion temperature. During the first $1.5 \mathrm{msec}$, ion temperatures are higher at the plasma edge than at the center.

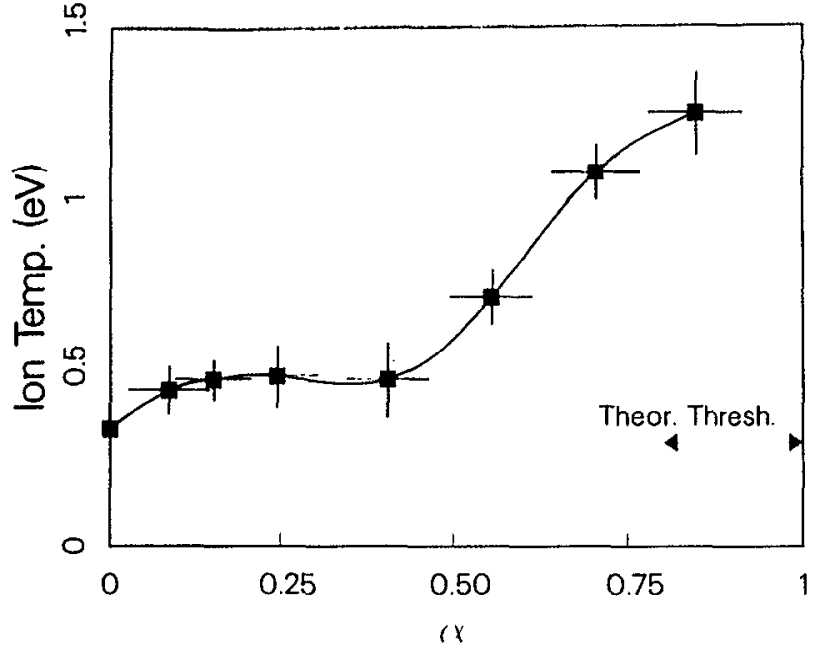

FIG. 14. Experimental values of $T_{i}$ plotted as a function of $\alpha$. The magnetic field was $300 \mathrm{G}$.

sured value of $1.25 \mathrm{eV}$. The fact that the threshold for heating is lowered from about 0.8 to 0.4 can be attributed to the more complicated geometry in this situation. As was touched upon in Sec. VI C, the dimension of the phase space was increased from three to five, allowing the ions to diffuse more easily throughout the phase space. Another interesting point is that for all the data points shown in Fig. 14 the electron temperature and space potential were identical to within $10 \%$. Therefore the ion temperature was independent of the mean electron temperature and mean space potential but was strongly dependent on the space potential fluctuation level.

\section{E. Evidence for nonadiabatic ion motion}

As was noted in Sec. VI C, the stochastic ion motion in large-amplitude electrostatic modes is nonconservative and an ion is free to attain very large (but not infinite) energies. The upper bound being given by Eq. (13). This implies that ions can be accelerated to energies far exceeding the potential that is due to the drift wave trough. The potential amplitude of the drift wave can be determined by means of Langmuir probes and this can be compared to the corresponding ion velocity distribution obtained using LIF. Electron temperature, electron density, and plasma potential were determined in the usual fashion. ${ }^{32}$ Here $T_{e}$ was determined from the I-V characteristic, $n_{e}$ was then determined from the ion saturation current, and, finally, the plasma potential was obtained by measuring the floating potential and then correcting for the temperature fluctuations,

$$
V_{s}-V_{f}=\frac{1}{2} T_{e} \ln \left(m_{i} / 2 \pi m_{e}\right) \text {. }
$$

Examples of the fluctuating plasma parameters are shown in Fig. 15. Thesc data werc taken using an 8 bit Transiac transient recorder at a digitization rate of $200 \mathrm{kHz}$. The data 


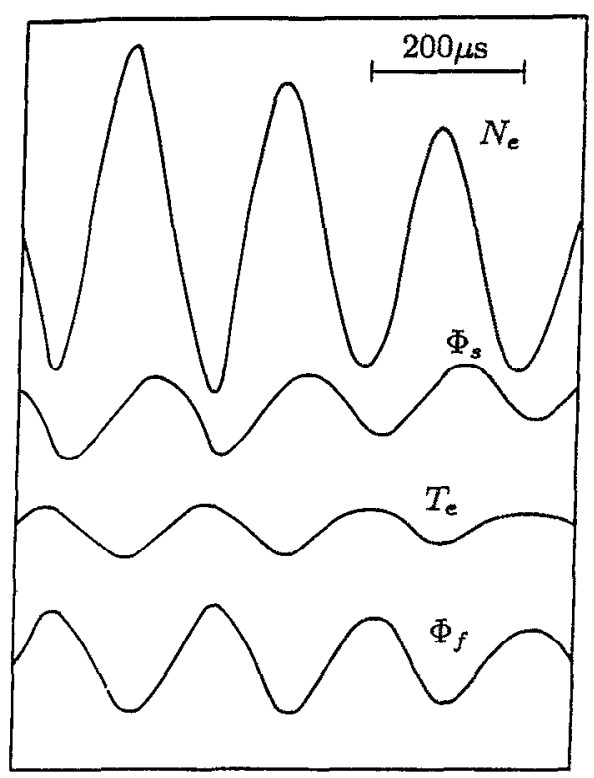

FIG. 15. Fluctuating plasma parameters measured by Langmuir probe. Here, 3000 plasma shots were required to obtain these data.

were averaged over 1000 plasma discharges to ensure precise plasma potential measurements. To maintain a constant phase reference, a separate Langmuir probe was used to trigger the data collection system. The duration of the sample shown is $200 \mu \mathrm{sec}$, which corresponds to a full wave period. By making these measurements at different radial positions in the plasma, it was possible to form a 2-D picture of the drift mode structure. Since the mode is rotating, the temporal fluctuations could be mapped into the poloidal angle of the plots. Figure 16 shows plots of density and plasma potential for a mode that is above the amplitude for stochastic behavior. The coherent nature of the modes and their large amplitude are evident from these plots. Figure 17 shows two ion distribution functions obtained for wave amplitudes below $(\alpha=0.40)$ and above $(\alpha=0.85)$ the thresholds for stochastic ion motion [Figs. 17(a) and 17(b), respectively]. The hatched regions indicate those ions that have energies greater than the peak to peak potential of the drift wave. Clearly, when the wave amplitude is above threshold, there are significant numbers of ions with energies considerably larger than the potential amplitude. Conversely, when the wave amplitude is below threshold, few ions have energies in excess of the potential amplitude of the wave. The existence of ions traveling at the sound speed was noted in the section on Landau damping of ion acoustic waves.

Attempts were made to use optical tagging to demonstrate nonconservative motion by showing that ions were being transported over the potential peak of the drift wave. However, this would have required the observation of ion transport over a distance of about $10 \mathrm{~cm}$. As described in Sec. IV, it was difficult to trace tagged particles over distances greater than $1-2 \mathrm{~cm}$. Only ions in the high-energy tail of the ion distribution could be traced for more than a few centimeters. It was possible, however, to perform velocity selection using tagging, as demonstrated in Sec. IV. The spa- (a)

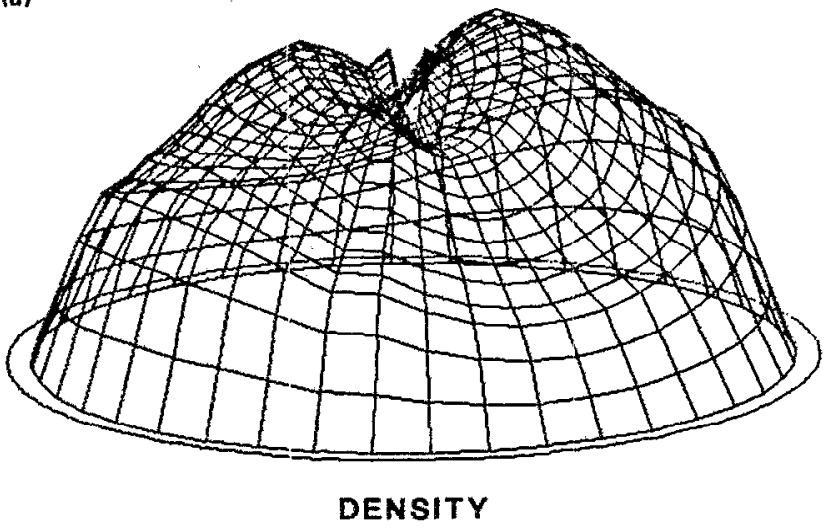

(b)

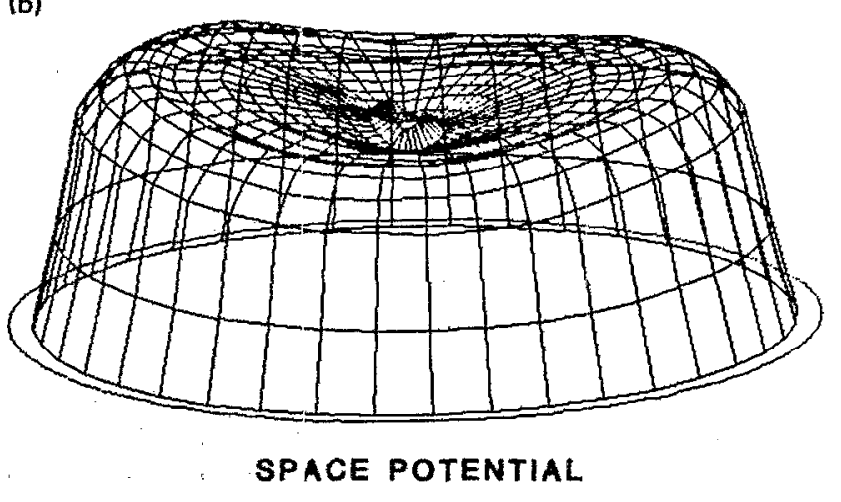

FIG. 16. Density and space potential plots for an $m=2$ mode. (a) Density (mean density $=5 \times 10^{11} \mathrm{~cm}^{-3}$ ). (b) Space potential (mean potential $=15 \mathrm{~V}$.

tial and temporal separation of the pump and search beams was such that only energetic ions could be tagged. In addition, the probing laser pulses were fired only in the vicinity of the potential peak of the drift wave. In this way it was possi-
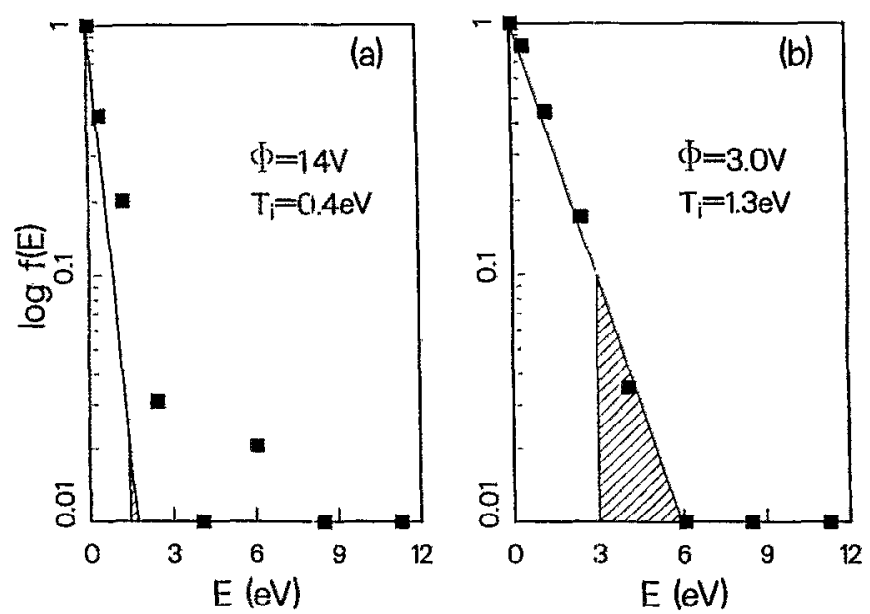

FIG. 17. Ion distribution functions (a) below $(\alpha=0.40)$ and (b) above $(\alpha=0,85)$ the threshold lor stochastic motion. The shaded regions represent those ions having energies greater than the peak-to-peak potential of the drift wave. The squares are the experimental data points and the solid line is the least-squares fit to the data. 

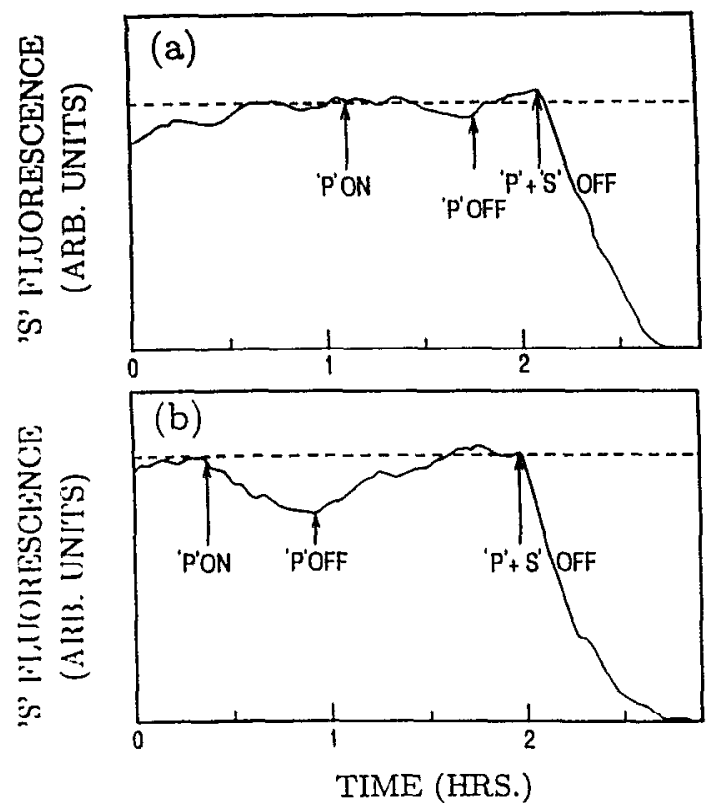

FIG. 18. (a) Optical tagging data that demonstrate that few ions have high energies when below the threshold for stochastic heating. (b) Optical tagging data that demonstrate the existence of high-energy ions when above the threshold for stochastic heating. The " $P$ " ON denotes the pump beam turn-on time. The " $P$ " OFF denotes the pump beam turn-off time and the $" P "+" S "$ OFF the pump and search beam turn-off time.

ble to observe energetic ions moving in regions of the space potential peaks. If the ion motion were, in fact, conservative, then ion energies should have been very low in these regions. A Langmuir probe was used to monitor the drift wave density fluctuations. Density fluctuations lead the space potential fluctuations by $\pi / 4$ (Fig. 9), allowing the position of the potential peak to be monitored. This reference probe was used to provide a $25 \mu \mathrm{sec}$ gate that triggered both the pump laser and the boxcar averager whenever the search laser pulse coincided with the wave potential peak. This method ensured that both the search and pump laser beams were triggered within $\pm \pi / 8$ of the wave peak. The search beam diameter was $3 \mathrm{~mm}$, while the pump beam was roughly 1.2 $\mathrm{cm}$ squarc. The minimum beam separation was set to be 4 $\mathrm{mm}$. The pump beam was made very much larger than the search beam in the hope that it would essentially swamp the search beam with particles and thereby enhance the tagging effect. The time interval between the pump (" $P$ ") and search (" $S$ ") laser pulses was $1.5 \mu \mathrm{sec}$, implying minimum particle velocities of $2.7 \times 10^{5} \mathrm{~cm} \mathrm{sec}^{-1}$ or energies of 1.5 $\mathrm{eV}$. Ion transport was examined both below and above the threshold for stochastic heating. Figure 18 shows the boxcar-averaged outputs of the search laser fluorescence signal as a function of time, both below Fig. 18(a) and above Fig. 18 (b), the threshold for strong ion heating. For case (a) the presence of the pump beam had little or no effect on the search beam fluorescence. For case (b), however, the pump beam reduced the search beam fluorescence signal was reduced by about $18 \%$, implying the existence of a large population of energetic ions possessing velocities in excess of $2.7 \times 10^{5} \mathrm{~cm} \mathrm{sec}^{-1}$ Very long averaging times were required
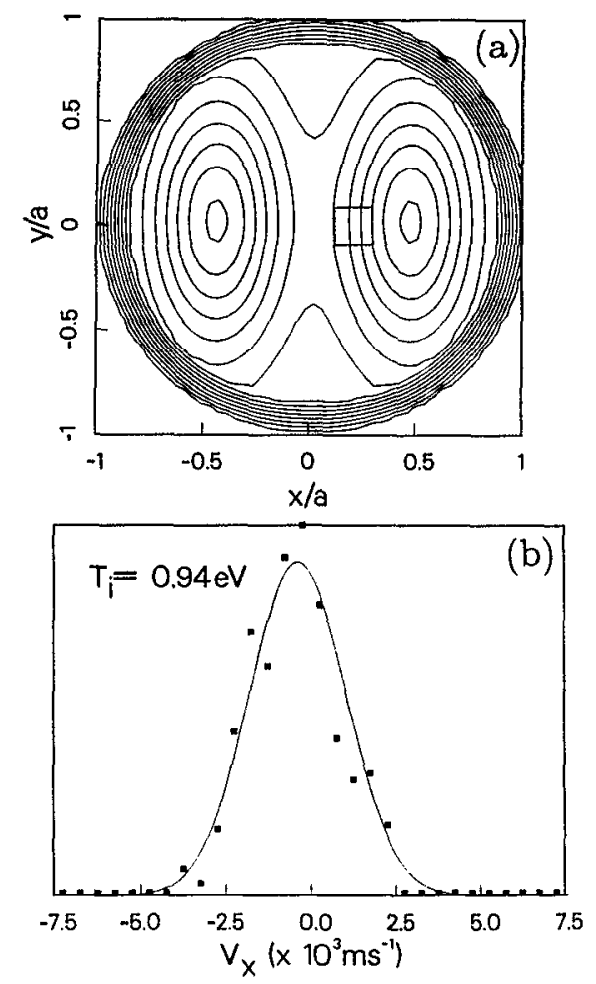

FIG. 19. (a) Contour plot of model drift wave space potential $(0.5 \mathrm{~V} / \mathrm{con}-$ tour). (b) Ion distribution function obtained by numerically integrating the orbits of $2 \times 10^{5}$ ions starting in the rotating potential shown in (a). Only those ions appearing in the small square box were used to determine the distribution function. For this distribution $T$, was $0.94 \mathrm{eV}$.

to achieve a good signal-to-noise ratio, because the pump laser had a low repetition rate.

\section{F. Numerical simulation of ion heating}

Stochastic ion heating was demonstrated by means of particle simulations. A two-dimensional electric field is now appropriate $(x \rightarrow r$ and $y \rightarrow \theta)$. The numerical calculations of heating performed to be described are identical to those performed in Sec. VI C, but the dimension of the phase space has been increased from three to five. The trajectories of $2 \times 10^{5}$ particles were calculated under the influence of the potential pattern shown in Fig. 19(a). The mode frequency, wave number, and wave amplitude were measured experimentally by means of a Langmuir probe and the toroidal field power supply was calibrated, so that the magnetic field was known. These experimentally determined parameters were then used in the numerical calculation. The parameters used were $B_{0}=400 \mathrm{G}, k_{\theta}=35.3 \mathrm{~m}^{-1}, v=5 \mathrm{kHz}$, and $\phi=3.5 \mathrm{~V}$ (peak to peak). The drift wave pattern was assumed to rotate at half the observed frequency. The wave was assumed to grow linearly from zero amplitude, at $t=0$, to the maximum amplitude, at $t=50 \mu \mathrm{sec}$. Ion motion along the magnetic field direction was ignored since for drift-Alfvén waves, $k_{1} \gg k_{\|}$. Only the toroidal magnetic field was considered, since this was much larger than the poloidal field. The toroidal field was assumed to have the usual $R{ }^{1} \mathrm{de}-$ pendence on the major radius. The ions were started from 
rest and their initial positions were normally distributed across the chamber. Any test ion striking the wall was assumed to be lost and then replaced by another test particle, which started life with zero velocity. Each ion trajectory was integrated, and the one-dimensional velocity distribution function was calculated every 0.025 of a gyroperiod. Only those ions found within the square region shown in Fig. 19 (a) were used to calculate the distribution function. This region had an area of $<1 \%$ of the total cross section of the torus, therefore there was no possibility of averaging over the phase of the wave when calculating the velocity distribution. An example of such a distribution function is shown in Fig. 19 (b). Figure 20 compares the heating results of the simulation described above to actual experimental heating results for an $m=2$ drift-Alfvén wave. The experimental measurements were made at the plasma center and the simulation measurements were made inside the square region shown in Fig. 19(a). The model underestimates the observed ion temperatures somewhat but reproduces the general trend of rapid heating and saturation of the ion temperature at about 1 $\mathrm{eV}$. The model assumed above was not self-consistent. However, since the electric and magnetic fields used in the calculations were measured experimentally, the model was felt to be useful. It is conceivable that a fully self-consistent calculation might alter the above results.

It is informative to examine the actual trajectories of individual ions under different conditions. Figure 21 (a) shows the calculated trajectory of an ion moving under the infuence of an electric field pattern of the type shown in Fig. 19(a). The inside wall of the chamber is to the left, and the magnetic field strength decreases from left to right across the chamber. For this calculation, $B_{0}=400 \mathrm{G}, k_{\theta}=31.7 \mathrm{~m}^{-1}$, $v=5 \mathrm{kHz}$, and $\phi=1.7 \mathrm{~V}$ (peak to peak), giving, $\alpha=0.22$. Figure 21 (b) shows the temporal variation of kinetic energy (normalized to the peak-to-peak potential amplitude) of this ion. The ion motion is regular and the kinetic energy oscillates sinusoidally between zero and some small amplitude as it gains and loses energy reversibly in the wave field.

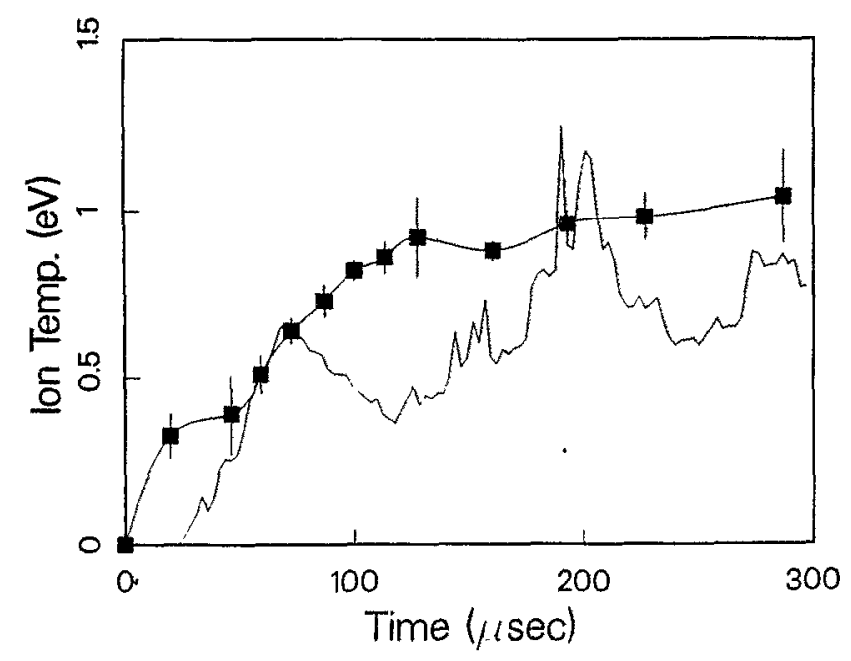

FIG. 20. Simulation of ion heating in the field of a drift wave. The solid lines represent the results of the simulation, while the individual data points represent the actual ion temperature measurements.

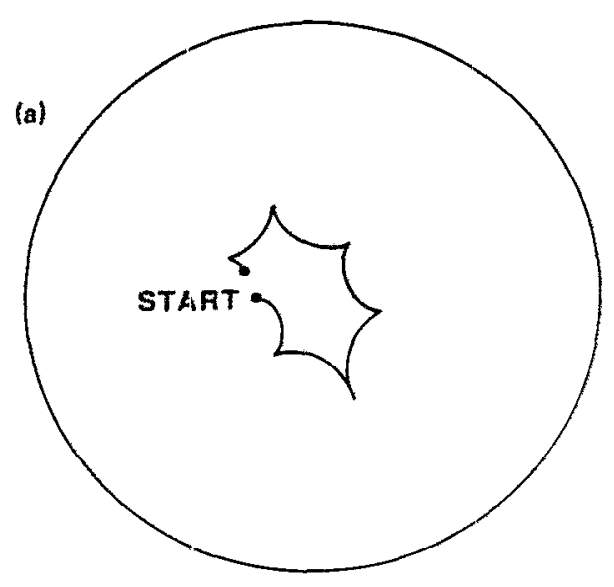

(b)

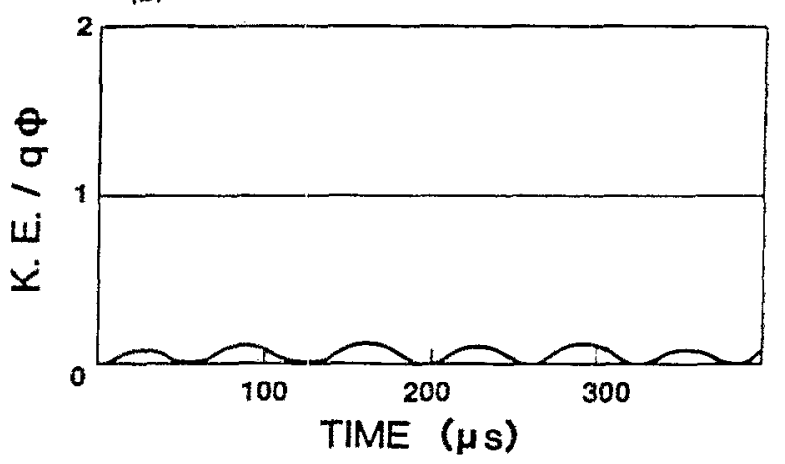

FIG. 21. (a) Typical calculated ion trajectory showing regular ion motion $(\alpha=0.22)$. (b) Kinetic energy of the same ion (normalized to maximum potential energy in the wave) as a function of time.

In Fig. 22 similar plcts show a test ion executing stochastic motion. The irregular nature of the ion motion is evident. In Fig. 22(b) the ion kinetic energy varies dramatically and randomly and often exceeds the peak-to-peak potential energy of the drift wave. The parameters used for this calculation are identical to those used in the first calculation, except that the potential amplitude was increased to $1.9 \mathrm{~V}$ (peak to peak), giving $\alpha=0.25$. The effect of this very small increase in wave potential is remarkable. The two preceding examples were carefully chosen to show the importance of initial conditions on the ion motion.

\section{ANOMALOUS ION HEATING IN RIFP'S}

Anomalously high ion temperatures and anomalously high rates of ion heating have been observed in all major, reversed-field pinch (RFP) experiments. ${ }^{8.9}$ For instance, Howell and Nagayama ${ }^{8}$ show that in ZT-40, ion temperatures exceded elcetron temperatures for sufficiently large plasma currents ( $T_{i} \approx 600 \mathrm{eV}, T_{e} \approx 450 \mathrm{eV}$, and $I>300 \mathrm{kA}$ ). Also, these ion temperatures were reached in $1 \mathrm{msec}$ or less, i.e., faster than would be possible via collisional energy exchange with electrons. More recently, anomalously high ion temperatures have been observed on the REPUTE device. ${ }^{9}$ For instance, $\mathrm{O}^{+4}$ temperatures were determined spectroscopically while deuterium and hydrogen temperatures were 


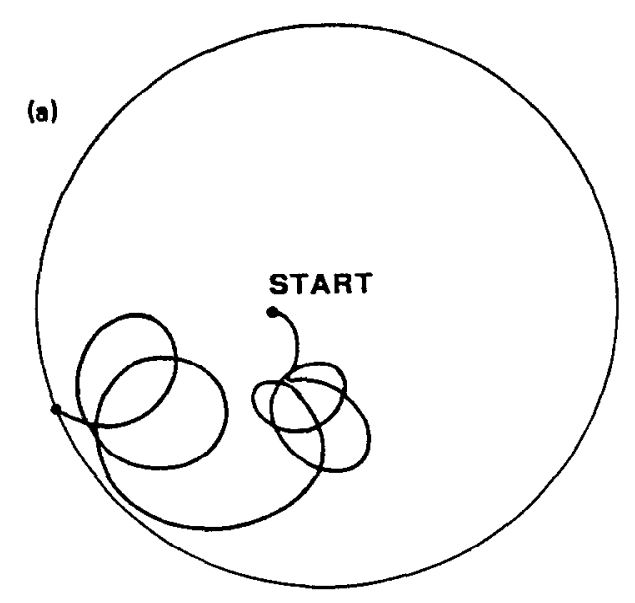

(b)

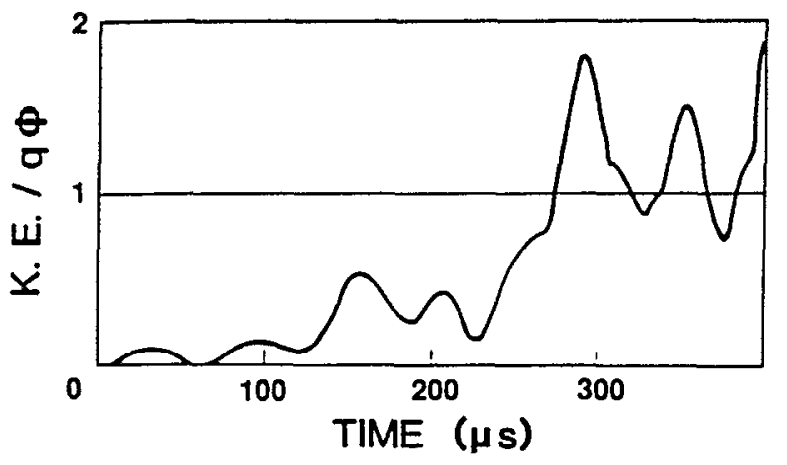

FIG. 22. (a) Typical calculated ion trajectory in tokamak minor cross section showing stochastic ion motion $(\alpha=0.25)$. (b) Kinetic energy of the same ion (normalized to maximum potential energy in the wave) as a function of time.

measured independently using charge exchange analysis. For $T_{e}=50 \mathrm{eV}$ ion temperatures were found to be $T_{i}\left(\mathrm{O}^{+4}\right)$ $=400 \mathrm{eV}, T_{i}\left(\mathrm{D}^{+}\right)=150 \mathrm{eV}$, and $T_{i}\left(\mathrm{H}^{+}\right)=100 \mathrm{eV}$.

Several authors have proposed the existence of currentdriven instabilities in RFP's. ${ }^{33,34}$ In particular, Gladd and Krall ${ }^{33}$ have shown drift waves to be strongly destabilized by the high currents found in RFP's. It is well known that the stability of drift waves is dependent upon the ratio of electron drift velocity to electron thermal velocity $\left(u / v_{e}\right)$. Typical values of $u / v_{e}$ for both Encore and ZT-40 are about 0.1, contrasting with a value of about 0.01 for a typical large tokamak such as PLT. In RFP's, ion heating has been observed to scale strongly with the ratio of plasma current to line density $(I / N),{ }^{8}$ and hence must depend strongly on electron drift velocity. For most tokamaks, ion heating is independent of $u / v_{e}$ and proceeds at a rate in accordance with the Spitzer relaxation time.

Few $\tilde{\phi}$ measurements have been made on RFP's. Some double-probe measurements were made on Zeta. ${ }^{35}$ These measurements showed $50-100 \mathrm{kHz}$ electric field fluctuations of $1000-3000 \mathrm{~V} \mathrm{~m}^{-1}$ and perpendicular correlation lengths $\lambda_{1} \sim 5 \mathrm{~cm}$ in deuterium discharges with $B \simeq 1000 \mathrm{G}$. Using typical parameters of $\lambda_{1}=0.05 \mathrm{~m}, B=0.05 \mathrm{~T}$, $E=2000 \mathrm{Vm}^{-1}, v<0.1$ and $\alpha=2.1$, and $T_{i}=33 \mathrm{eV} \sim T_{e}$. So it is possible that stochastic heating by electrostatic insta- bilities is responsible for the high ion temperatures observed in RFP's. Another important point to note is the very rapid nature of the acceleration process. For the parameters described above heating will occur in about $5 \mu \mathrm{sec}$. This is less than a wave period. Therefore the wave need not be coherent and long lived. A short-lived impulse can cause strong local heating as long as it exists for longer than $\tau \sim \alpha / \omega$. It is also worth noting that $\alpha$ depends linearly on $m_{i} / Z$, and so impurity ions will be stochastically heated at faster rates than hydrogen or deuterium majority ions but may then heat these majority ions via ion-ion collisions. Also, spuriously high ion temperatures will be inferred from spectroscopic measurements of the linewidths of heavy impurity ions if they have not come into equilibrium with the bulk plasma.

\section{DISCUSSION}

The techniques of laser-induced fluorescence (LIF) and optical tagging (OT) have been successfully applied to Q-machine and discharge plasmas for several years. The initial purpose of the present work was to extend these powerful techniques to more complex plasma devices. The LIF technique was successfully used to determine ion temperatures and the OT technique was then used to demonstrate ion gyrorotation.

LIF was then used to demonstrate that ion heating in the Encore tokamak was anomalously fast. Various possible ion heating mechanisms were considered but were found to be inadequate. The ions appeared to be heated by a largeamplitude, saturated, drift-Alfvén waves that satisfied the condition $\alpha=m_{i} k_{\perp}^{2} \phi_{0} / q B_{0}^{2} \leqslant 1$. Evidence was presented that indicated that the ion motion became stochastic in the presence of these modes, resulting in strong ion heating. It appears likely that energy transfer from the wave to the ions can provide a possible saturation mechanism. However, the exact nature of the saturation mechanism was unknown and other mechanisms might have been at work, and these could have caused the observed ion heating.

Until recently, it has been implicitly assumed that there are only two categories of heating mechanisms at work in nonturbulent plasmas, namely, collisions with energetic particles and resonant wave-particle interactions. The theoretical work of several authors ${ }^{23-25}$ has nonresonant wave-particle interactions can also cause heating. When $\alpha \leqslant 1$, the ion excursion due to the polarization drift becomes comparable to the wavelength of the mode. At this point the guidingcenter approximation breaks down and the ion motion becomes stochastic. Calculations showed that the condition $\alpha \lesssim 1$ held regardless of the wave frequency, as long as $\omega<\omega_{c i}$. (For the case $\omega \gg \omega_{c i}$, the condition $\alpha \gtrsim 4$ was required. ${ }^{24}$ ) It was also shown that stochastic ion heating for these conditions is related to ion trapping.

The drift-Alfvén modes were current driven and the wave amplitude could be controlled by carefully varying the plasma current. In this manner it was possible to observe the transition from adiabatic to stochastic motion. As expected from the numerical results of Sec. VI, this transition was quite abrupt. As a check on the LIF temperature measurements Landau damping of ion acoustic waves was measured 
as a function of $T_{c} / T_{i}$. These measurements proved that the LIF-determined ion temperatures were valid and not merely an artifact of the averaging procedure. It was shown that the ion motion was nonadiabatic, by showing that significant numbers of ions possessed kinetic energies that werc in excess of their maximum possible potential energies in the field of the drift wave. The existence of these very energetic ions was predicted from the Poincare surface of section plots presented in Sec. VI. From these plots it was also evident that ions could not be accelerated to arbitrarily high energies, and the peak ion energy was given by Eq. (13). A particle simulation of magnetized ions subjected to a low-frequency electrostatic mode reproduced the observed heating rate and peak ion temperature. An essentially Maxwellian velocity distribution was obtained without the mediation of collisions.

Anomalously high ion temperatures and heating rates have been observed on all RFP major experiments. From the limited measurements of electrostatic fluctuation measurements that are available it appears very likely that the heating mechanism described in the present work is responsible for these effects in RFP's.

\section{ACKNOWLEDGMENTS}

This work was taken from the doctoral dissertation of one of the authors (J. M. McC.). One of us (R. A. S.) would like to thank the Sherman Fairchild Foundation for a fellowship. The authors would like to thank Mr. F. Cosso for his assistance with the electronics.

This work was supported by National Science Foundation Grant No. PHY8312489.

\section{APPENDIX: DESTABILIZATION OF DRIFT WAVES}

Linear drift waves are destabilized by the existence of a phase difference between the density and space potential fluctuations. In collisional plasmas this phase difference is introduced by electron-ion collisions, in collisionless plasmas by electron Landau damping by resonant thermal electrons or by an electron drift parallel to the magnetic field. When the electron mean-free path is less than the wavelength collisional damping effects predominate. The parallel wave number is given by $k_{z} \simeq(q R)^{-1}$, where $q$ is the safety factor and $R$ is the major radius. So the condition for collisionless damping is $v_{e i} q R / v_{e}<1$. For typical Encore parameters $\left(q \sim 2, R=38.1 \mathrm{~cm}, v_{e}=1.5 \times 10^{8} \mathrm{~cm} \mathrm{sec}^{-1}, v_{e i}\right.$ $\left.=8.4 \times 10^{5} \mathrm{sec}^{-1}\right) v_{e i} q R / v_{e} \sim 0.4$ and the drift waves are in the collisionless regime. In order to determine whether or not the axial current has a significant destabilizing effect, it is necessary to look at the growth rate of the instability in detail. The frequency and growth rate of current-driven drift waves are given by ${ }^{21}$

$$
\begin{aligned}
& \omega_{r}=\omega_{e}^{*} /\left(1+k_{y}^{2} \rho_{s}^{2}\right), \\
& \omega_{i}=\frac{\sqrt{\pi} \omega^{2}}{k_{z} v_{e}\left(1+k_{y}^{2} \rho_{s}^{2}\right)}\left(k_{y}^{2} \rho_{s}^{2}+\frac{k_{z} v_{0}}{\omega}\right),
\end{aligned}
$$

where

$$
\rho_{s}=\frac{c_{s}}{\omega_{c i}}, \quad \omega_{e}^{*}:=-\frac{k_{y} T_{e}}{m_{e} \omega_{c e}} \frac{d \ln n}{d x},
$$

and where $k_{z}$ is the parallel wave number, $k_{y}$ is the perpendicular wave number, and $v_{0}$ is the parallel, electron drift velocity. The first term in brackets in $\mathrm{Eq}$. (A2) represents the contribution of Landau damping to the growth rate, while the second term represents the contribution of the axial current. Typical parameters for Encore are $k_{z} \simeq(q R)^{-1}$ $\sim 0.01 \mathrm{~cm}^{-1}, k_{y}=\mathrm{m} / \mathrm{r} \sim 0.3 \mathrm{~cm}^{-1}, v_{0} \sim 3 \times 10^{7} \mathrm{~cm} \mathrm{sec}^{-1}$, and $\rho_{s} \sim 5 \mathrm{~cm}$. Inserting these values into Eq. (A2) shows that the second term is about an order of magnitude larger than the first term and that the drift waves are strongly current driven.

${ }^{1}$ R. M. Measures, J. Appl. Phys. 39, 5232 (1968).

${ }^{2}$ D. Dimock, E. Hinnov, and L. C. Johnson, Phys. Fluids 12, 1730 (1969).

${ }^{3}$ R. A. Stern and J. A. Johnson, III, Phys. Rev. Lett. 34, 1548 (1975).

"V. S. Burakov, P. Ya. Misyakov, P. A. Naumenko, S. V. Nechaev, G. T. Razdobarin, V. V. Semenov, L. V. Sokolova, and I. P. Folomkin, JETP Lett. 26, 403 (1977).

${ }^{5}$ C. H. Muller, III and K. H. Burrell, Phys. Rev. Lett. 47, 330 (1981).

' J. M. McChesney, R. A. Stern, and P. M. Bellan, Phys. Rev. Lett. 59, 1436 (1987).

${ }^{7}$ R. A. Stern, D. N. Hill, and N. Rynn, Phys. Lett A 93, 127 (1983).

${ }^{8}$ R. B. Howell and $Y$. Naizayama, Phys. Fluids 28, 743 (1985).

${ }^{9}$ T. Fujita, K. Saito, J. Matsui, Y. Kamada, H. Morimoto, Z. Yoshida, and N. Inoue, Nucl. Fusion 31, 3 (1991).

${ }^{10}$ H. P. Summers and R. W. P. McWhirter, J. Phys. B: Atom. Mol. Phys. 12, 2387 (1979).

"C. A. Moore, G. P. Davis, and R. A. Gottscho, Phys. Rev. Lett. 52, 538 (1984).

${ }^{12}$ W. P. West, D. M. Thomas, E. S. Ensberg, J. S. DeGrassie, and J. F. Baur, Rev. Sci. Instrum. 57, 1552 (1986).

${ }^{13}$ R. Koslover and R. Mclvilliams, Rev. Sci. Instrum. 57, 2421 (1986).

${ }^{14}$ H. R. Griem, Plasma Spectroscopy (McGraw-Hill, New York, 1964).

${ }^{15}$ W. Lotz, Z. Phys. 216, 241 (1968).

${ }^{16}$ B. A. Trubnikov, Rev. Plasma Phys. 1, 105 (196.5).

${ }^{17}$ F. Anderegg, R. A. Stern, F. Skiff, B. A. Hammel, M. Q. Tran, P. J. Paris, and P. Kohler. Phys. Rev, Lett. 57, 329 (1986).

${ }^{18}$ D. R. Dakin, T. Tajima, G. Benford, and N. Rynn, J. Plasma Phys. 15, 175 (1976).

${ }^{19}$ S. Q. Mah, H. M. Skarsgard, and A. R. Strilchuk, Phys. Rev. Lett. 25, 1409 (1970).

${ }^{20}$ R. Hatakeyama, M. Oet tl, E. Märk, and R. Schrittwieser, Phys. Fluids 23, 1774 (1980).

${ }^{21}$ A. B. Mikhailovskii and L. I. Rudakov, JETP 17.621 (1963).

${ }^{22}$ E. D. Fredrickson and P. M. Bellan, Phys. Fluids 28, 1866 (1985).

${ }^{23}$ G. R. Smith and A. N. Kaufman, Phys. Rev. Lett. 34, 1613 (1975).

${ }^{24}$ C. F. F. Karney, Phys. Fluids 21, 1584 (1978).

${ }^{25}$ J. F. Drake and T. T. Lee, Phys. Fluids 24, 1115 (1981).

${ }^{26}$ F. Doveil, Phys. Rey, Leitt. 46, 532 (1981).

${ }^{27}$ F. Skiff, F. Anderegg, and M. Q. Tran, Phys. Rev. Lett. 58, 1430 (1987).

${ }^{28} \mathrm{C}$. Z. Cheng and H. Okuda, Nucl. Fusion 18, 587 (1978).

${ }^{29}$ L. Chen and C. K. Birdsall, Phys. Fluids 16, 2229 (1973).

${ }^{30}$ A. A. Chernikov, R. Z. Sagdeev, D. A. Usikov, M. Yu Zakharov, and G. M. Zasiavsky, Nature 326, 559 (1987).

${ }^{31}$ N. A. Krall and A. W. Trivelpiece, Principies of Plasma Physics (McGraw-Hill, New Ycrk, 1973).

${ }^{32}$ F. F. Chen, in Plasma Liagnostic Techniques, edited by R. H. Huddlestone and S. L. Leonard (Academic, New York, 1965), p. 113.

${ }^{33}$ N. T. Gladd and N. A. Krall, Phys. Fluids 29, 1640 (1986),

${ }^{34}$ T. E. Cayton, Phys. Fluids 28, 598 (1985).

${ }^{35}$ D. C. Robinson and M. G. Rusbridge, Phys. Fluids 14, 2499 (1971). 